\title{
Kernels with Prescribed Surface Tension \& Mobility for Threshold Dynamics Schemes
}

\author{
Selim Esedoğlu* \\ Matt Jacobs ${ }^{\dagger}$ \\ University of Michigan \\ University of Michigan \\ Pengbo Zhang $\ddagger$ \\ University of Michigan
}

February 5, 2017

\begin{abstract}
We show how to construct a convolution kernel that has a desired anisotropic surface tension and desired anisotropic mobility to be used in threshold dynamics schemes for simulating weighted motion by mean curvature of interfaces, including networks of them, in both two and three dimensions. Moreover, we discuss necessary and sufficient conditions for the positivity of the kernel which, in the case of two-phase flow, ensures that the resulting scheme respects a comparison principle and implies convergence to the viscosity solution of the level set formulation of the flow. In particular, we show, in a barrier-type statement, that the kernel cannot possibly be positive unless both the mobility and the surface tension satisfy necessary conditions in three dimensions, and give a complete characterization. Among other results is a threshold dynamics scheme that is guaranteed to dissipate a nonlocal approximation to the interfacial energy in the fully anisotropic, multiphase setting, using the new kernel construction.
\end{abstract}

Keywords: Multi-phase flow, curvature motion, threshold dynamics

\section{Introduction}

Originally proposed by Merriman, Bence, and Osher (MBO) in [33, 32], threshold dynamics - also known as diffusion or convolution generated

${ }^{*}$ Corresponding author. esedoglu@umich.edu. 530 Church St. Ann Arbor, MI 48105

${ }^{\dagger}$ majaco@umich.edu. 530 Church St. Ann Arbor, MI 48105

${ }^{\ddagger}$ pbzhang@umich.edu. 530 Church St. Ann Arbor, MI 48105

(C) 2017. This manuscript version is made available under the Elsevier user license http://www.elsevier.com/open-access/userlicense/1.0/ 
motion - is a very efficient algorithm for approximating the motion by mean curvature of an interface. The algorithm generates a discrete in time approximation to mean curvature motion by alternating two very simple steps: convolution with a kernel, and pointwise thresholding. In its simplest form (for isotropic, two-phase mean curvature flow), it generates the approximate solutions $\Sigma^{k}$ at time $t=k \delta t$ from an initial condition $\Sigma^{0} \subset \mathbb{R}^{d}$ as follows:

Algorithm:(MBO'92): Given a time step size $\delta t>0$, alternate the following steps:

1. Convolution:

$$
\psi^{k}=\frac{1}{(\delta t)^{\frac{d}{2}}} K\left(\frac{x}{\sqrt{\delta t}}\right) * \mathbf{1}_{\Sigma^{k}}
$$

2. Thresholding:

$$
\Sigma^{k+1}=\left\{x: \psi^{k}(x) \geq \frac{1}{2} \int_{\mathbb{R}^{d}} K d x\right\} .
$$

The convolution kernel $K: \mathbb{R}^{d} \rightarrow \mathbb{R}$ was chosen in [33] to be the Gaussian

$$
K(x)=\frac{1}{(4 \pi)^{\frac{d}{2}}} \exp \left(-\frac{|x|^{2}}{4}\right) .
$$

but the possibility of choosing other kernels is also mentioned in [32]. With $K$ given by 3 ), the boundary of the set $\partial \Sigma^{k}$ can be shown to evolve, to leading order, by mean curvature motion; see e.g. [39] for a truncation error analysis, and e.g. [16], [27] for proofs of convergence. Among the benefits of Algorithm (1) \& (2) are are 1. implicit representation of the interface as in the phase field or level set methods, allowing for graceful handling of topological changes, 2. unconditional stability, where the time step size is restricted only by accuracy considerations, and 3. very low per time step cost when implemented on uniform grids.

Motion by mean curvature arises as $L^{2}$ gradient descent, in an appropriate sense, for perimeter of sets, which in turn appears in variational models for a great variety of applications. These range from image processing and computer vision (e.g. the Mumford-Shah model [36] for image segmentation) to materials science (e.g. Mullins' model [35] for grain boundary motion in polycrystals). More recently, such variational models and their minimization via gradient descent have also been applied in the context of machine learning and artificial intelligence (e.g. graph partitioning models for supervised clustering of data [17]). The MBO scheme, its variants, and its extensions have attracted sustained interest in the context of each one of these ap- 
plications. In this paper, we will focus on applications in materials science, where anisotropic and multiphase versions of mean curvature motion play a central role.

The question of whether threshold dynamics algorithm (1) \& (2) can be extended to anisotropic curvature flows, by appropriate choice of the convolution kernel $K$ in (1), has been the topic of numerous investigations in the literature; a summary is given in Section 3. The present paper is devoted to providing a decisive, constructive answer to this question, by showing how to choose the kernel $K$ given a desired possibly anisotropic surface tension and possibly anisotropic mobility for the interface. Combined with new multiphase versions of threshold dynamics recenty proposed in [14], the kernel constructions of this paper yield unconditionally stable schemes for the weighted mean curvature flow of a general $n$-phase network by allowing $n$-choose- 2 anistropic surface tensions and $n$-choose-2 anisotropic mobilities (one pair for each interface in the network) to be individually specified. This full level of generality is also a first for threshold dynamics schemes.

\section{Preliminaries and Notation}

We will be concerned with possibly anisotropic interfacial energies defined on partitions of a domain $D$. $D$ will typically be the $d$-dimensional annulus, i.e. a cube in $\mathbb{R}^{d}$ with periodic boundary conditions. By a partition of $D$, we mean a prescribed number $N$ of sets $\Sigma_{1}, \ldots, \Sigma_{N} \subseteq D$ that satisfy

$$
\bigcup_{i=1}^{N} \Sigma_{i}=D \text { and } \Sigma_{i} \cap \Sigma_{j}=\left(\partial \Sigma_{i}\right) \cap\left(\partial \Sigma_{j}\right) \text { for } i \neq j
$$

Let $H^{s}$ denote the $s$-dimensional Hausdorff measure on $D$. Given a non-negative, continuous, even function $\sigma: \mathbb{S}^{d-1} \rightarrow \mathbb{R}^{+}$with $\sigma(x)>0$ for $x \neq 0$, we first consider the two-phase surface energy

$$
E(\Sigma, \sigma)=\int_{\partial \Sigma} \sigma(n(x)) d H^{d-1}
$$

where $n(x)$ denotes the outward unit normal to $\partial \Sigma$. We will also consider the multiphase extension of energy (5) to partitions. Let $N \in \mathbb{N}^{+}$ denote the number of phases, and define the set of distinct pairs of indices:

$$
\mathcal{I}_{N}=\{(i, j) \in\{1, \ldots, N\} \times\{1, \ldots, N\}: i \neq j\} .
$$

Our multiphase energy is:

$$
E(\boldsymbol{\Sigma}, \sigma)=\sum_{(i, j) \in \mathcal{I}_{N}} \int_{\left(\partial \Sigma_{i}\right) \cap\left(\partial \Sigma_{j}\right)} \sigma_{i, j}(n(x)) d H^{d-1}(x)
$$


where we write $\boldsymbol{\Sigma}=\left(\Sigma_{1}, \ldots, \Sigma_{N}\right)$. It will be convenient to assume that $\sigma$ has been extended to $\sigma: \mathbb{R}^{d} \rightarrow \mathbb{R}^{+}$as

$$
\sigma(x)=|x| \sigma\left(\frac{x}{|x|}\right) \text { for } x \neq 0
$$

so that it is positively 1 -homogeneous. We will assume that $\sigma$ is then a convex function on $\mathbb{R}^{d}$; this condition will ensure well-posedness of the two-phase energy (5). Define the unit ball (i.e. the Frank diagram) $B_{\sigma}$ of $\sigma$ as

$$
B_{\sigma}=\{x: \sigma(x) \leq 1\}
$$

which is thus a closed, convex, centrally symmetric set. We will further require $B_{\sigma}$ to be smooth and strongly convex: There exists $R$ such that $B_{\sigma}$ is supported by balls of radius $R$ that contain it. In particular, we stay clear of the crystalline cases (where $B_{\sigma}$ is a polytope) except via approximation. In two dimensions, we will also write $\sigma=\sigma(\theta)$, where $\theta$ is the angle that the unit normal makes with the positive $x_{1}$-axis. In that case, strong convexity of $B_{\sigma}$ is equivalent to the condition

$$
\sigma^{\prime \prime}(\theta)+\sigma(\theta)>0 .
$$

Given a set $\Omega \subset \mathbb{R}^{d}$, its support function $h_{\Omega}$ is defined to be

$$
h_{\Omega}(x)=\sup _{y \in \Omega} x \cdot y .
$$

Note the simple but useful fact

$$
h_{\Omega_{1}+\Omega_{2}}=h_{\Omega_{1}}+h_{\Omega_{2}}
$$

where $\Omega_{1}+\Omega_{2}$ denotes the Minkowski sum of the sets $\Omega_{1}$ and $\Omega_{2}$ :

$$
\Omega_{1}+\Omega_{2}=\left\{x: x=x_{1}+x_{2} \text { with } x_{1} \in \Omega_{1} \text { and } x_{2} \in \Omega_{2}\right\}
$$

i.e. it is just the dilation of $\Omega_{1}$ by $\Omega_{2}$. The Wulff shape $W_{\sigma}$ associated with the anisotropy $\sigma$ is defined as

$$
W_{\sigma}=\left\{y: \sup _{x \in B_{\sigma}} x \cdot y \leq 1\right\} .
$$

i.e. it is the unit ball of the support function $h_{B_{\sigma}}$ of $B_{\sigma}$; in yet other words, it is the unit ball of the dual norm to $\sigma$. The sets $B_{\sigma}$ can therefore in turn be obtained from $W_{\sigma}$ by the formula

$$
B_{\sigma}=\left\{x: \sup _{y \in W_{\sigma}} x \cdot y \leq 1\right\},
$$

exhibiting the well known duality between $B_{\sigma}$ and $W_{\sigma}$. Our assumptions on $B_{\sigma}$ imply that $W_{\sigma}$ is also strongly convex and has smooth boundary. 
We will denote the spherical Radon transform of an even function $f: \mathbb{S}^{d-1} \rightarrow \mathbb{R}$ by

$$
\mathcal{J}_{s} f(n)=\int_{\mathbb{S}^{d-1} \cap n^{\perp}} f(x) d H^{d-1}(x) .
$$

Closely connected with the spherical Radon transform $\mathcal{J}_{s}$ is the cosine transform $\mathcal{T}$, also defined on even functions on the sphere, as follows:

$$
\mathcal{T} f(n)=\int_{\mathbb{S}^{d-1}} f(x)|x \cdot n| d H^{d-1}(x) .
$$

The relation between $\mathcal{J}_{s}$ and $\mathcal{T}$ is given by

$$
\square \mathcal{T}=\mathcal{T} \square=\mathcal{J}_{s}
$$

where $\square=\Delta_{\mathbb{S}^{d-1}}-(d-1) I$ and $\Delta_{\mathbb{S}^{d-1}}$ denotes the surface Laplacian (i.e. the Laplace-Beltrami operator) on $\mathbb{S}^{d-1}$. The operators $\mathcal{J}_{s}, \mathcal{T}$, and of course $\square$ are symmetric, in the sense that

$$
\int_{\mathbb{S}^{d-1}} \phi \mathcal{L} \psi d H^{d-1}=\int_{\mathbb{S}^{d-1}} \psi \mathcal{L} \phi d H^{d-1}
$$

for $\mathcal{L} \in\left\{\mathcal{J}_{s}, \mathcal{T}, \square\right\}$, where $\phi$ and $\psi$ are any two smooth functions on $\mathbb{S}^{d-1}$. They also commute with one another. For a given anisotropy $\sigma$ with a strongly convex $B_{\sigma}$ and smooth $\partial B_{\sigma}$, its generating function $\omega: \mathbb{S}^{d-1} \rightarrow \mathbb{R}$ is defined to be the following even function:

$$
\omega:=\mathcal{T}^{-1} \sigma=\square \mathcal{J}_{s}^{-1} \sigma=\mathcal{J}_{s}^{-1} \square \sigma .
$$

For $d=2$, the expression (16) for the generating function of an anisotropy simplifies to

$$
\omega(\theta)=\frac{1}{4}\left\{\sigma^{\prime \prime}\left(\theta-\frac{\pi}{2}\right)+\sigma\left(\theta-\frac{\pi}{2}\right)\right\}
$$

where $\theta$ denotes the polar angle of an $x \in \mathbb{S}^{1}$; see e.g. [13]. We will use the following definition of the Fourier transform on $\mathbb{R}^{d}$ :

$$
\widehat{f}(\xi)=\int_{\mathbb{R}^{d}} f(x) e^{-i x \cdot \xi} d \xi \text { so that } f(x)=\frac{1}{(2 \pi)^{d}} \int_{\mathbb{R}^{d}} \widehat{f}(\xi) e^{i \xi \cdot x} d \xi
$$

for e.g. $f$ in Schwartz class.

For $d=2$ or 3 , we will study approximations for $L^{2}$ gradient flow of energies (5) and (7), which are known as weighted mean curvature flow (of an interface and a network). The normal speed of an interface in three dimensions under this flow is given by

$$
\begin{aligned}
v_{\perp}(x)=\mu(n(x))\left(\left(\partial_{s_{1}}^{2} \sigma(n(x))\right.\right. & +\sigma(n(x))) \kappa_{1}(x) \\
+ & \left.\left(\partial_{s_{2}}^{2} \sigma(n(x))+\sigma(n(x))\right) \kappa_{2}(x)\right)
\end{aligned}
$$


where $\kappa_{1}$ and $\kappa_{2}$ are the two principal curvatures, and $\partial_{s_{i}}$ denotes differentiation along the great circle on $\mathbb{S}^{2}$ that passes through $n(x)$ and has as its tangent the $i$-th principal curvature direction. In two dimensions, the expression simplifies to

$$
v_{\perp}(x)=\mu(n(x))\left(\sigma^{\prime \prime}(n(x))+\sigma(n(x))\right) \kappa(x) .
$$

While materials science literature e.g. $[12,24]$ appears to allow the mobility factor $\mu: \mathbb{S}^{d-1} \rightarrow \mathbb{R}^{+}$in (18) or (19) to be any positive function of the normal, a natural and important subclass of mobilities are those $\mu$ that have a convex one-homogeneous extension (as in (8)) to $\mathbb{R}^{d}$. Indeed, as explained in [6], motion law (18) arises as gradient descent for energy (5) with respect to a norm $\mu: \mathbb{R}^{d} \rightarrow \mathbb{R}$ on normal vector fields on $\partial \Sigma$ e.g. via the well-known discrete-in-time minimizing movements [11] procedure of Almgren, Taylor \& Wang [2], and independently, Luckhaus \& Sturzenhecker [29]:

$$
\Sigma^{k+1}=\underset{\Sigma}{\arg \min }\left\{E(\Sigma, \sigma)+\frac{1}{\delta t} \int_{\Sigma \Delta \Sigma^{k}} d_{\Sigma^{k}}^{\mu^{*}}(x) d x\right\}
$$

where $d_{\Sigma^{k}}^{\mu^{*}}$ denotes the distance function to the interface $\partial \Sigma^{k}$ at the $k$-th time step, with respect to the dual norm $\mu^{*}$ of the norm $\mu$ :

$$
\mu^{*}(x)=\sup _{y: \mu(y) \leq 1} x \cdot y .
$$

It is the unique viscosity solution of the Hamilton-Jacobi equation

$$
\begin{aligned}
\mu^{2}(\nabla u) & =1 \text { for } x \in\left(\partial \Sigma^{k}\right)^{c}, \\
u & =0 \text { for } x \in \partial \Sigma^{k}
\end{aligned}
$$

which can be used to verify consistency of scheme (20). The set of convex mobilities $\mu$ thus constitute a distinguished subclass and will be the subject of particular attention in Section 4.1.2.

In addition to (18), a condition known as the Herring angle condition [23] holds along triple junctions: For $d=3$, at a junction formed by the meeting of the three phases $\Sigma_{i}, \Sigma_{j}$, and $\Sigma_{k}$, this condition reads

$$
\begin{array}{r}
\left(\ell \times n_{i, j}\right) \sigma_{i, j}\left(n_{i, j}\right)+\left(\ell \times n_{j, k}\right) \sigma_{j, k}\left(n_{j, k}\right)+\left(\ell \times n_{k, i}\right) \sigma_{k, i}\left(n_{k, i}\right) \\
+n_{j, i} \sigma_{i, j}^{\prime}\left(n_{i, j}\right)+n_{k, j} \sigma_{j, k}^{\prime}\left(n_{j, k}\right)+n_{i, k} \sigma_{k, i}^{\prime}\left(n_{k, i}\right)=0
\end{array}
$$

where $n_{i, j}$ is the unit normal vector to the interface $\left(\partial \Sigma_{i}\right) \cap\left(\partial \Sigma_{j}\right)$ pointing in the $\Sigma_{i}$ to $\Sigma_{j}$ direction, $\ell=n_{j, k} \times n_{i, j}$ is a unit vector tangent to the triple junction, and $\sigma_{i, j}^{\prime}\left(n_{i, j}\right)$ denotes derivative of $\sigma_{i, j}$ taken on $\mathbb{S}^{2}$ in the direction of the vector $\ell \times n_{i, j}$. In the isotropic 
setting, (22) simplifies to the following more familiar form, known as Young's law:

$$
\sigma_{i, j} n_{i, j}+\sigma_{j, k} n_{j, k}+\sigma_{k, i} n_{k, i}=0 .
$$

Finally, we note that well-posedness (lower semi-continuity) of the multiphase energy (7) in its full generality is a complicated matter [3]. At the very least, the surface tensions $\sigma_{i, j}: \mathbb{R}^{d} \rightarrow \mathbb{R}^{+}$need to be convex and satisfy a pointwise triangle inequality

$$
\sigma_{i, j}(n)+\sigma_{j, k}(n) \geq \sigma_{i, k}(n)
$$

for all distinct $i, j$, and $k$, and all $n \in \mathbb{S}^{d-1}$. In case the $\sigma_{i, j}$ are positive constants, (24) is known to be also sufficient for well-posedness of model (7).

The variational model (4) \& (7) on partitions and the dynamics (19) subject to (22) it generates, play a central role in models of microstructural evolution in polycrystalline materials starting with the work [35] of Mullins. Such materials are very common; they include, for instance, most metals and ceramics. Each phase $\Sigma_{i}$ in (4) represents a microscopic single crystal piece of the material, called a grain, distinguished from its neighbors only by its differing crystalographic orientation. The mismatch in crystal orientations across grain boundaries $\partial \Sigma_{i} \cap \partial \Sigma_{j}$ contribute to the excess internal energy (7) which is dissipated through a reconfiguration of the microstructure when the material is annealed (held at high temperature). The shapes and sizes of the grains have dramatic implications for physical properties, such as conductivity and yield strength, of a polycrystalline material. For an introduction to this application, including derivations of the relevant formulas recalled in this section, see e.g. [26], [21], [42]. Background on the transforms (12) and (13) quoted above and their significance in convex geometry can be found in e.g. [20], [7], [22].

\section{Previous Work}

Generalizations of Merriman, Bence, and Osher's Algorithm (1) \& (2) to anisotropic surface energies have been considered in a number of works in the existing literature. The basic idea is to take the convolution kernel $K$ to be a more general kernel (than the Gaussian) satisfying the properties

$$
K(x) \in L^{1}\left(\mathbb{R}^{d}\right), x K(x) \in L^{1}\left(\mathbb{R}^{d}\right), \text { and } K(x)=K(-x) .
$$

together with

$$
\int_{\mathbb{R}^{d}} K(x) d x>0
$$


For convenience, from now on we will write

$$
K_{\varepsilon}(x)=\frac{1}{\varepsilon^{d}} K\left(\frac{x}{\varepsilon}\right)
$$

for the rescaled versions of a given convolution kernel $K$.

One of the first contributions to the study of Algorithm (1) \& (2) with general convolution kernels is by Ishii, Pires, and Souganidis [27], who establish the convergence of the algorithm to the viscosity solution of the equation

$$
u_{t}=F\left(D^{2} u, D u\right)
$$

where

$$
F(M, p)=\left(\int_{p^{\perp}} K(x) d H^{d-1}(x)\right)^{-1}\left(\frac{1}{2} \int_{p^{\perp}}\langle M x, x\rangle K(x) d H^{d-1}(x)\right)
$$

for $p \in \mathbb{R}^{d}$ and $M$ a $d \times d$ symmetric matrix, provided that $K$ is a positive convolution kernel with certain additional decay and continuity properties. Positivity of the kernel is required for the scheme to preserve the comparison principle that applies to underlying interfacial motion, and is essential for the viscosity solutions approach taken in [27]. On the other hand, the consistency calculation given in the paper applies to more general (e.g. sign changing) kernels (and also appears in [39] for the special case of a Gaussian). This study extends to the case of anisotropic curvature motions earlier proofs of convergence appearing in [16] and [4] for the isotropic version of the scheme that uses the Gaussian as the convolution kernel.

The paper by Ishii et. al. does not address the inverse problem of constructing a convolution kernel for a given surface tension (and possibly a mobility), which is perhaps the more practical problem from a numerical methods perspective. The first contribution in this direction is by Ruuth \& Merriman in [40], who propose a construction in $2 D$. Given an anisotropy $f:[0,2 \pi] \rightarrow \mathbb{R}^{+}$, the focus of the authors is to construct a kernel (characteristic function of a judiciously chosen star shaped domain) that, when used in threshold dynamics, would generate a normal speed of the form

$$
v_{\perp}(x)=\left(f^{\prime \prime}(\theta)+f(\theta)\right) \kappa(x)
$$

where $\theta$ is the angle that the unit outer normal $n(x)$ at $x$ makes with the positive $x$-axis. This approach conflates the contributions to $v_{\perp}$ in (19) from mobility and surface tension factors. Indeed, there are infinitely many surface tension \& mobility pairs $(\sigma, \mu)$ that correspond to the same $f$ and hence the same normal speed in (29); the discussion in [40] does not elucidate the two factors. This is a particularly important matter in multiphase flows, since surface tensions determine the 
equilibrium condition at junctions according to (22). And in fact, it turns out that for Ruuth \& Merriman's construction, the corresponding surface tension is not given by $f$ in (29); see [13] for a detailed discussion. On the plus side, these kernels are positive (being characteristic functions) and thus preserve the comparison principle.

More recently, Bonnetier et. al. [8] have proposed a construction that works in both $2 \mathrm{D}$ and $3 \mathrm{D}$. The Fourier transform of their kernels is explicit in terms of the surface tension:

$$
\widehat{K}(\xi)=\exp \left(-\sigma^{2}(\xi)\right)
$$

It turns out that the corresponding mobility satisfies $\mu:=\sigma$ identically, see [13]. This construction often yields non-positive kernels, even in two dimensions, preventing the authors from giving a rigorous proof of convergence. Moreover, as soon as the anisotropy $\sigma$ does not have an ellipsoid as its unit ball, (30) has a singularity at the origin, leading to slow decay of $K$. We will revisit this construction in Section 4.2 and remedy some of its shortcomings.

In [15], a variational formulation for the original MBO scheme (1) \& (2) was given. In particular, it was shown that the following functional defined on sets, with kernel $K$ chosen to be the Gaussian $G$, which had previously been established $[1,34]$ to be a non-local approximation to (isotropic) perimeter, is dissipated by the MBO scheme at every step, regardless of time step size:

$$
E_{\sqrt{\delta t}}\left(\Sigma, K_{\sqrt{\delta t}}\right)=\frac{1}{\sqrt{\delta t}} \int_{\Sigma^{c}} K_{\sqrt{\delta t}} * \mathbf{1}_{\Sigma} d x
$$

Thus, (31) is a Lyapunov functional for algorithm (1) \& (2), establishing its unconditional gradient stability. Moreover, the following minimizing movements [2, 29] interpretation involving (31) for algorithm (1) \& (2) was given in [15]:

$\Sigma^{k+1}=\underset{\Sigma}{\arg \min } E_{\sqrt{\delta t}}\left(\Sigma, K_{\sqrt{\delta t}}\right)+\frac{1}{\sqrt{\delta t}} \int\left(\mathbf{1}_{\Sigma}-\mathbf{1}_{\Sigma^{k}}\right) K_{\sqrt{\delta t}} *\left(\mathbf{1}_{\Sigma}-\mathbf{1}_{\Sigma^{k}}\right) d x$

where the kernel $K$ was again taken to be $G$. In [15], variational formulation (31) \& (32) was also extended to the multiphase setting.

Let us recall the following fact from [15] that ensures (31) is a Lyapunov functional for scheme (1) \& (2), establishing the connection between the variational problem (31) and threshold dynamics, and underlining the significance of $\widehat{K}$ :

Proposition 1 (from [15]) Let $K$ satisfy (25) and (26). If $\widehat{K} \geq 0$, threshold dynamics algorithm (1) \& (2) decreases energy (31) at every time step, regardless of the time step size. 
In [15], the variational formulation (32) was then extended to the multiphase energy (7) in case the surface tensions $\sigma_{i, j}$ are constant but possibly distinct:

$$
E(\boldsymbol{\Sigma}, \sigma)=\sum_{(i, j) \in \mathcal{I}_{N}} \sigma_{i, j} H^{d-1}\left(\partial \Sigma_{i} \cap \partial \Sigma_{j}\right)
$$

in which case the Lyapunov functional becomes

$$
E_{\sqrt{\delta t}}\left(\boldsymbol{\Sigma}, K_{\sqrt{\delta t}}\right)=\frac{1}{\sqrt{\delta t}} \sum_{(i, j) \in \mathcal{I}_{N}} \sigma_{i, j} \int_{\Sigma_{j}} K_{\sqrt{\delta t}} * \mathbf{1}_{\Sigma_{i}} d x
$$

We also consider a relaxation of (34):

$$
E_{\sqrt{\delta t}}\left(\mathbf{u}, K_{\sqrt{\delta t}}\right)=\frac{1}{\sqrt{\delta t}} \sum_{(i, j) \in \mathcal{I}_{N}} \sigma_{i, j} \int_{D} u_{j} K_{\sqrt{\delta t}} * u_{i} d x
$$

over the following convex set of functions satisfying a box constraint:

$$
\mathcal{K}=\left\{\mathbf{u} \in L^{1}\left(D,[0,1]^{N}\right): \sum_{i=1}^{N} u_{i}(x)=1 \text { a.e. } x \in D\right\} .
$$

There is a corresponding minimizing movements scheme that can be derived from (35) that leads to the following extension of threshold dynamics to the constant but possibly unequal surface tension multiphase energy (33):

Algorithm: (from [15]): Given a time step size $\delta t>0$, alternate the following steps:

1. Convolution:

$$
\psi_{i}^{k}=K_{\sqrt{\delta t}} * \sum_{j \neq i} \sigma_{i, j} \mathbf{1}_{\Sigma_{j}^{k}}
$$

2. Thresholding:

$$
\Sigma_{i}^{k+1}=\left\{x: \psi_{i}^{k}(x) \leq \min _{j \neq i} \psi_{j}^{k}(x)\right\} .
$$

Various conditions are provided in [15] for ensuring that Algorithm (37) $\&(38)$ is unconditionally gradient stable (decreases energy (34) for any $\delta t>0)$. The question turns out to be interesting, with connections to isometric embeddability of finite metric spaces into Euclidean spaces. In particular, the triangle inequality $(24)$ on $\sigma_{i, j}$ appears to be neither necessary nor sufficient.

It turns out that the minimizing movements interpretation is especially helpful in the anisotropic setting, as it allows identifying easily 
a normal dependent surface tension and a normal dependent mobility factor associated with a given convolution kernel; moreover, this understanding can then be extended to the full multiphase, anisotropic setting. We recall the following facts from [13] in this connection:

Proposition 2 (from [13]) Let $\Sigma$ be a compact subset of $\mathbb{R}^{d}$ with smooth boundary. Let $K: \mathbb{R}^{d} \rightarrow \mathbb{R}$ be a kernel satisfying (25). Then:

$$
\lim _{\delta t \rightarrow 0^{+}} E_{\sqrt{\delta t}}\left(\Sigma, K_{\sqrt{\delta t}}\right)=\int_{\partial \Sigma} \sigma_{K}(n(x)) d H^{d-1}(x)
$$

where the surface tension $\sigma_{K}: \mathbb{R}^{d} \rightarrow \mathbb{R}^{+}$is defined as

$$
\sigma_{K}(n):=\frac{1}{2} \int_{\mathbb{R}^{d}}|n \cdot x| K(x) d x .
$$

A stronger, Gamma convergence version of Proposition 2 is given in [14] for a class of kernels that include sign changing ones. In polar coordinates, the expression for the surface tension $\sigma_{K}$ that corresponds to a given convolution kernel $K$ is:

$$
\sigma_{K}(n)=\frac{1}{2} \int_{0}^{\infty} r^{d} \int_{\mathbb{S}^{d-1}}|n \cdot x| K(r x) d H^{d-1}(x) d r .
$$

We should also note, as is done in [13], that equations (16) \& (40) imply the generating function $\omega_{K}$ of the anisotropy $\sigma_{K}$ that corresponds to a given kernel $K$ is given by:

$$
\omega_{K}(n):=\frac{1}{2} \int_{0}^{\infty} K(r n) r^{d} d r .
$$

In [13], the following expression is obtained for the mobility $\mu_{K}$ associated with a given kernel $K$ :

$$
\frac{1}{\mu_{K}(n)}:=\int_{n^{\perp}} K(x) d H^{d-1}(x) .
$$

Equation (42) can alternatively be written using the spherical Radon transform $\mathcal{J}_{s}$ :

$$
\frac{1}{\mu_{K}}=\mathcal{J}_{s} \int_{0}^{\infty} K(r n) r^{d-2} d r .
$$

Also in [13], the following alternative expressions for $\sigma_{K}$ and $\mu_{K}$ in terms of the Fourier transform $\widehat{K}$ of the convolution kernel $K$ are provided:

$$
\begin{aligned}
& \sigma_{K}(n)=-\frac{1}{2 \pi} \text { F. P. } \int_{\mathbb{R}} \frac{\widehat{K}(n \xi)}{\xi^{2}} d \xi, \text { and } \\
& \mu_{K}(n)=2 \pi\left(\int_{\mathbb{R}} \widehat{K}(n \xi) d \xi\right)^{-1} .
\end{aligned}
$$


We also need the following Barrier Theorem from [13] that places a restriction on the positivity of convolution kernels in terms of the Wulff shape $W_{\sigma}$ of the given anisotropy $\sigma$.

Theorem 1 (from [13]) Threshold dynamics algorithm (1) \& (2) with a positive kernel $K$ can approximate weighted mean curvature flow (18) associated with an anisotropic surface tension $\sigma: \mathbb{S}^{d-1} \rightarrow \mathbb{R}$ (for some choice of mobility $\mu: \mathbb{S}^{d-1} \rightarrow \mathbb{R}$ ) if and only if the corresponding Wulff shape $W_{\sigma}$ is a zonoid.

Let us briefly recall zonoids - also known as projection bodies. They are centrally symmetric convex bodies that are limits, in the Hausdorff topology, of zonotopes, which are defined as (finite) vector sums of line segments. In $\mathbb{R}^{d}$, a convex polytope with nonempty interior is a zonotope if and only if every $d-1$ dimensional face of it is a zonotope. Thus, for $d=2$, any centrally symmetric, convex body is a zonoid. For $d=3$, this is no longer the case: a convex polytope is a zonotope only if its faces are centrally symmetric polygons, and the closure of such polytopes forms a strict subset of centrally symmetric convex bodies. A simple example of a non-zonoid in $\mathbb{R}^{3}$ is the octahedron. Moreover, there exists a neighborhood of the octahedron that contains no zonoids. Theorem 1 implies that there is no monotone threshold dynamics scheme for an anisotropy $\sigma$ the Wulff shape $W_{\sigma}$ of which falls into such a neighborhood, even though $W_{\sigma}$ may be smooth and strongly convex. See $[20,7]$ for these facts and much more information about zonoids.

Finally, we recall from [14] that simple modifications to the original threshold dynamics algorithm (1) \& (2) may allow relaxing the conditions on the convolution kernel and its Fourier transform without sacrificing some of the desirable properties discussed above. For example, the following single growth version, introduced in [14], possesses the energy dissipation property described in Proposition 1 for the much larger class of convolution kernels that can be expressed as a sum of a positive kernel and one with a positive Fourier transform:

$$
K=K^{1}+K^{2} \text { with } K^{1} \geq 0 \text { and } \widehat{K^{2}} \geq 0 .
$$

It entails two convolutions per time step vs. one for the original scheme: 
Algorithm 3: (from [14]): Given a time step size $\delta t>0$, alternate the following steps:

1. First convolution:

$$
\psi^{k}=K_{\sqrt{\delta t}} * \mathbf{1}_{\Sigma^{k}} .
$$

2. First thresholding:

$$
\Sigma^{k+\frac{1}{2}}=\left\{x: \psi^{k}(x) \geq \frac{1}{2} \int_{\mathbb{R}^{d}} K d x\right\} \cup \Sigma^{k} .
$$

3. Second convolution:

$$
\psi^{k+\frac{1}{2}}=K_{\sqrt{\delta t}} * \mathbf{1}_{\Sigma^{k+\frac{1}{2}}} .
$$

4. Second thresholding:

$$
\Sigma^{k}=\Sigma^{k+\frac{1}{2}} \backslash\left\{x: \psi^{k+\frac{1}{2}}(x) \leq \frac{1}{2} \int_{\mathbb{R}^{d}} K d x\right\} .
$$

In addition to dissipating energy (34) for a much wider variety of convolution kernels, Algorithm (45)-(48) is monotone for positive kernels, and thus can be shown to converge to the viscosity solution of the corresponding geometric evolution (27) in this case; see [14].

Extensions of the multiphase, isotropic, unequal surface tension Algorithm (37) \& (38) of [15] to the fully anisotropic, multiphase setting of energy (7) are in principle easy to come up with, and have been described in [13] and [14]. The analogue of the Lyapunov functional (34) in this setting, i.e. the non-local approximation to (7), is simply

$$
E_{\sqrt{\delta t}}\left(\boldsymbol{\Sigma}, \mathbf{K}_{\sqrt{\delta t}}\right)=\frac{1}{\sqrt{\delta t}} \sum_{(i, j) \in \mathcal{I}_{N}} \int_{\Sigma_{i}}\left(K_{i, j}\right)_{\sqrt{\delta t}} * \mathbf{1}_{\Sigma_{j}} d x
$$

where each component $K_{i, j}$ of the collection of kernels $\mathbf{K}$ satisfies

$$
\frac{1}{2} \int_{\mathbb{R}^{d}}|n \cdot x| K_{i, j}(x) d x=\sigma_{i, j} .
$$

One of the main contributions of this paper, presented in Section 4, shows how to construct well-behaved kernels $K$ that satisfy (50) while having prescribed, possibly anisotropic mobilities. The most straightforward, immediate extension of algorithm (37) \& (38) to energy (49) is Algorithm (89) \& (90) that is recalled from [13] in the Appendix. It works well in practice, as shown in numerical experiments in [13] as well as in Section 5.2 of this work. However, verifying rigorously that it dissipates energy (49) for a wide enough class of surface tensions 
to be of interest in applications appears to be difficult. On the other hand, Algorithm (45)-(48) can also be extended to the fully anisotropic, multiphase setting of energy (7) while ensuring dissipation of the corresponding Lyapunov functional (49) for essentially all surface tensions of interest in applications. This extension, Algorithm (91)-(95) recalled in the Appendix from [14], is demonstrated and compared to the simpler Algorithm (89) \& (90) on the numerical experiments of Section 5.2 using the new kernels constructed in Section 4 of this paper.

\section{The New Convolution Kernels}

In this main section of the paper, we present two new constructions of a convolution kernel $K$ for a given, possibly anisotropic, target surface tension $\sigma_{*}: \mathbb{S}^{d-1} \rightarrow \mathbb{R}^{+}$and target mobility $\mu_{*}: \mathbb{S}^{d-1} \rightarrow \mathbb{R}^{+}$. Both two and three dimensions are addressed. Both constructions identify the mobility and surface tension factors, and are therefore suitable for use in the multiphase setting.

The first construction, presented in Section 4.1, yields smooth, compactly supported kernels that are positive, so that scheme (1) \& (2) preserves the monotonicity (comparison principle) of the underlying evolution (18). As already implied by the barrier Theorem 1, there is necessarily a difference between two and three dimensions in this endeavor. Our results give a fairly complete picture of when this goal can be accomplished, and how to do it. These kernels do not necessarily have positive Fourier transforms, but the variant (45)-(48) of threshold dynamics in the two-phase, and (91)-(95) in the multi-phase setting, ensure dissipation of the corresponding energy (31) and (34).

The second construction, presented in Section 4.2, is a variant of the construction of [8] and is the more general: It allows any convex surface tension $\sigma_{*}$ and any positive mobility $\mu_{*}$, both in two and three dimensions, and yields a convolution kernel $K$ the Fourier transform $\widehat{K}$ of which is positive: $\widehat{K} \geq 0$. Moreover, unlike in [8], the resulting kernel is always Schwartz class. Threshold dynamics scheme (1) \& (2) using such a kernel thus dissipates the non-local interfacial energy (31) according to Proposition 1. However, as in [8], these kernels may not be positive, even in two dimensions.

\subsection{Positive Kernels}

In this section, we present new, positive convolution kernels for possibly anisotropic, desired surface tension and mobility pairs $\left(\sigma_{*}, \mu_{*}\right)$, leading to monotone threshold dynamics schemes. There are significant differences in two and three dimensions, so these two cases are discussed separately below. Proposition 3 in Section 4.1.1 yields positive, 
compactly supported, and smooth convolution kernels for essentially any anisotropic surface tension and mobility pair in two dimensions. In three dimensions, Lemma 1 and Proposition 4 of Section 4.1.2 essentially classify all anisotropic surface tension and mobility pairs for which such a kernel can be found, and for all such cases exhibit the desired kernels explicitly. Let us summarize the implication of these constructions by the following immediate consequence of results from [27] and [13]:

Corollary 1 In two dimensions, given essentially any anisotropic surface tension and mobility pair, a convolution kernel can be found such that two-phase threshold dynamics algorithms (1) \& (2) and (45)-(48), when extended to bounded uniformly continuous functions in the standard manner, converge to the viscosity solution of the corresponding evolution (18) on any finite time interval. Proposition 3 exhibits these kernels.

In three dimensions, there are surface tension and mobility pairs for which a monotone threshold dynamics scheme cannot be constructed. For essentially all those for which it can, Proposition 4 exhibits kernels with which the extension of algorithms (1) \&3 (2) and (45)-(48) to uniformly continuous functions will again converge to the viscosity solution of the corresponding evolution.

Our approach is as follows: Given $\left(\sigma_{*}, \mu_{*}\right)$, according to (39) and (42), we will need to solve the following coupled system of integral equations:

$$
\left\{\begin{aligned}
\int_{\mathbb{R}^{d}} K(x)|n \cdot x| d x & =\sigma_{*}(n), \text { and } \\
\int_{n^{\perp}} K(x) d H^{d-1}(x) & =\frac{1}{\mu_{*}(n)}
\end{aligned}\right.
$$

for the unknown function $K$. Note that there is in fact vast nonuniqueness of the solution, so it may be possible to impose additional conditions (besides positivity).

System (51) can be more conveniently expressed using the cosine and spherical Radon transforms as in formulas (41) \& (43):

$$
\left\{\begin{aligned}
\int_{0}^{\infty} K(r n) r^{d} d r & =\omega_{*}(n):=\mathcal{T}^{-1} \sigma_{*}(n), \text { and } \\
\int_{0}^{\infty} K(r n) r^{d-2} d r & =\mathcal{J}_{s}^{-1}\left[\frac{1}{\mu_{*}}\right](n) .
\end{aligned}\right.
$$

For both $d=2$ and $d=3$, the essential idea is the following: Formulas (39) \& (42) indicate that surface tension and mobility of a kernel vary differently as the convolution kernel is dilated along radial directions. We exploit this simple observation. With that in mind, let $\eta: \mathbb{R} \rightarrow \mathbb{R}$ 
be the following compactly supported, smooth, positive bump function:

$$
\eta(x)= \begin{cases}\exp \left(-\frac{1}{x^{2}(x-2)^{2}}\right) & \text { if } x \in(0,2), \\ 0 & \text { otherwise }\end{cases}
$$

For $j \in \mathbb{N}^{+}$, let

$$
m_{j}=\int_{0}^{2} x^{j} \eta(x) d x
$$

denote its moments.

\subsubsection{Positive Kernels in 2D}

In two dimensions, it turns out that a positive, smooth, compactly supported convolution kernel that is strictly positive at the origin can be constructed for any given surface tension \& mobility pair $\left(\sigma_{*}, \mu_{*}\right)$ that satisfies the following minimal assumptions:

(1.1) $B_{\sigma_{*}}$ is strongly convex and $\partial B_{\sigma_{*}}$ is smooth,

(1.2) $\mu_{*}: \mathbb{S}^{1} \rightarrow \mathbb{R}^{+} \backslash\{0\}$ is smooth.

We have

Proposition 3 Under conditions (1.1) \& (1.2) on $\sigma_{*}$ and $\mu_{*}$, there exists a positive, smooth, compactly supported convolution kernel $K$ : $\mathbb{R}^{2} \rightarrow \mathbb{R}^{+}$such that $\sigma_{K}=\sigma_{*}$ and $\mu_{K}=\mu_{*}$.

Proof: To solve the system (51), we look for a kernel $K$ that in polar coordinates has the form

$$
K(r, \theta)=\alpha(\theta) \eta(\beta(\theta) r)
$$

where $\alpha, \beta: \mathbb{R} \rightarrow \mathbb{R}^{+}$are $\pi$-periodic, smooth functions. Substituting (55) into (52) gives

$$
\begin{aligned}
\frac{\alpha(\theta)}{\beta^{3}(\theta)} m_{2} & =\omega_{*}(\theta), \text { and } \\
\frac{\alpha(\theta)}{\beta(\theta)} m_{0} & =\mathcal{J}_{s}^{-1}\left[\frac{1}{\mu_{*}}\right](\theta) .
\end{aligned}
$$

Note that for $d=2$,

$$
\mathcal{J}_{s}^{-1}\left[\frac{1}{\mu_{*}}\right](\theta)=\frac{1}{\mu_{*}\left(\theta-\frac{\pi}{2}\right)}
$$

and by (17),

$$
\omega_{*}(\theta)=\frac{1}{4}\left\{\sigma_{*}^{\prime \prime}\left(\theta-\frac{\pi}{2}\right)+\sigma_{*}\left(\theta-\frac{\pi}{2}\right)\right\} .
$$


Note that by our assumption on $B_{\sigma_{*}}$ above, $\omega_{*}(\theta)>0$ on $\theta \in[0,2 \pi]$. Solving system (56) for $\alpha$ and $\beta$ and using (57) \& (58) gives

$$
\begin{aligned}
& \alpha(\theta)=\left(\frac{4 m_{2}}{m_{0}^{3} \mu_{*}^{3}\left(\theta-\frac{\pi}{2}\right)\left[\sigma_{*}^{\prime \prime}\left(\theta-\frac{\pi}{2}\right)+\sigma_{*}\left(\theta-\frac{\pi}{2}\right)\right]}\right)^{\frac{1}{2}} \\
& \beta(\theta)=\left(\frac{4 m_{2}}{m_{0} \mu_{*}\left(\theta-\frac{\pi}{2}\right)\left[\sigma_{*}^{\prime \prime}\left(\theta-\frac{\pi}{2}\right)+\sigma_{*}\left(\theta-\frac{\pi}{2}\right)\right]}\right)^{\frac{1}{2}}
\end{aligned}
$$

Formulas (55) \& (59) give an explicit prescription for the convolution kernel in terms of the desired surface tension $\sigma_{*}$ and mobility $\mu_{*}$.

Remark: The kernel can be easily modified to be strictly positive at the origin: Replace $\left(\sigma_{*}, \mu_{*}\right)$ in the construction with $\left(\tilde{\sigma}_{*}, \tilde{\mu}_{*}\right)$ where

$$
\tilde{\sigma}_{*}(x)=\sigma(x)-\varepsilon \text { and } \frac{1}{\tilde{\mu}_{*}(x)}=\frac{1}{\mu_{*}(x)}-\varepsilon
$$

and $\varepsilon>0$ is chosen small enough so that $B_{\tilde{\sigma}}$ is strongly convex and $\tilde{\mu}_{*}>0$. Denote the resulting kernel $\tilde{K}$. Then, the kernel $K=\tilde{K}+$ $\exp \left(-\varepsilon|x|^{2}\right)$ satisfies $\sigma_{K}=\sigma_{*}$ and $\mu_{K}=\mu_{*}$.

\subsubsection{Positive Kernels in 3D}

The situation is more complicated in three dimensions. The essential question is positivity of the right hand sides of the system of integral equations (52) that entail the inverse cosine and inverse spherical Radon transforms. It turns out that such positivity questions are intimately connected with long studied problems and certain mathematical objects arising in convex geometry. This connection with convex geometry literature was already noted and utilized in [13] in formulating its barrier Theorem 1 quoted in Section 3. That theorem says that a necessary condition for a positive convolution kernel to be found for the target anisotropy $\sigma_{*}: \mathbb{S}^{2} \rightarrow \mathbb{R}^{+}$(regardless of the mobility) is that the corresponding Wulff shape $W_{\sigma_{*}}$ be a zonoid (also known as a projection body), an important class of centrally symmetric convex bodies that appear prominently in the convex geometry literature; see Section 3 for a brief discussion and e.g. [7, 20] for much more.

When we confront the question of simultaneously achieving both a target zonoidal surface tension $\sigma_{*}$ and a target mobility $\mu_{*}$ with a positive convolution kernel, a related class of objects, known as intersection bodies [30], and their connections to a widely studied problem known as the Busemann-Petty problem [10], again from convex geometry, come into play. In what follows, we will need to appeal to the resolution of this problem in [19] for the case $d=3$. 
Our first result in this direction is the barrier type Lemma 1 below. It states that in order to accommodate a wide enough class of mobilities $\mu_{*}$ using positive convolution kernels, we need to demand more from $W_{\sigma_{*}}$ than just being a zonoid. The issue is that there are strongly convex and smooth zonoids the generating functions $\omega_{*}$ of which vanish somewhere on $\mathbb{S}^{2}$. If $\omega_{*}$ corresponding to $\sigma_{*}$ vanishes even at a single point, however, it turns out $\mu_{*}$ cannot arise from the gradient descent formulation (20) of the interfacial motion (18):

Lemma 1 Let $\sigma_{*}: \mathbb{R}^{3} \rightarrow \mathbb{R}^{+}$be an anisotropy such that $W_{\sigma_{*}}$ is a smooth and strongly convex zonoid. If $K$ is a positive convolution kernel such that $\sigma_{K}=\sigma_{*}$ and its corresponding mobility $\mu_{K}: \mathbb{S}^{2} \rightarrow$ $\mathbb{R}^{+} \backslash\{0\}$ is smooth with a convex one-homogeneous extension to $\mathbb{R}^{3}$, then $W_{\sigma_{*}}$ can be written as the Minkowski sum of a zonoid and a sphere.

In particular, a threshold dynamics scheme that is consistent with an evolution law (18) arising from the gradient descent formulation (20) cannot possibly be monotone unless $W_{\sigma_{*}}$ is the dilation of a zonoid by a sphere.

Proof: By hypothesis, $\mu_{K}=\mu_{*}$ extends as a norm to $\mathbb{R}^{3}$. Therefore, $\frac{1}{\mu_{*}}$ is the radial function of a convex body in $\mathbb{R}^{3}$. By Theorem 5.2 and its Corollary 5.3 in [19], we have

$$
\mathcal{J}_{s}^{-1}\left[\frac{1}{\mu_{*}}\right]>0
$$

on $\mathbb{S}^{2}$. (Close inspection of Theorem 5.1 in [19] indicates inequality (60) is strict under our assumptions on $\mu$ ). Then, (43) implies that

$$
\int_{0}^{\infty} K(r x) r d r>0 \text { for all } x \in \mathbb{S}^{2} .
$$

But then, we also have

$$
\omega_{*}(x)=\omega_{K}(x)=\int_{0}^{\infty} K(r x) r^{3} d r>0 \text { for all } x \in \mathbb{S}^{2} .
$$

Let $\varepsilon=\frac{1}{2} \min _{x \in \mathbb{S}^{2}} \omega_{*}(x)>0$, and define the new anisotropy

$$
\sigma_{*}^{\prime}(n)=\int_{\mathbb{S}^{2}}\left(\omega_{*}(x)-\varepsilon\right)|x \cdot n| d H^{2}(x) .
$$

Then, we can write

$$
\sigma_{*}(x)=\sigma_{*}^{\prime}(x)+\varepsilon|x|
$$

so that by (10),

$$
h_{W_{\sigma_{*}}}=h_{W_{\sigma_{*}^{\prime}}}+h_{W_{\varepsilon|x|}} \text {. }
$$


That implies

$$
W_{\sigma_{*}}=W_{\sigma_{*}^{\prime}}+W_{\varepsilon|x|} \cdot
$$

$W_{\sigma_{*}^{\prime}}$ is a zonoid since its generating function is $\omega_{*}-\varepsilon \geq 0$, and $W_{\varepsilon|x|}$ is a sphere.

Lemma 1 motivates placing the assumption on $\sigma_{*}$ that $W_{\sigma_{*}}$ be the dilation of a zonoid by a sphere, which we will adopt for the rest of this section. This is a dense subset of zonoids in the Hausdorff metric. As another difference of three dimensions from two, it turns out that even with this stronger assumption on $\sigma_{*}$, the mobility factor $\mu_{*}$ in a given target surface tension \& mobility pair $\left(\sigma_{*}, \mu_{*}\right)$ needs to satisfy certain additional necessary conditions in order for there to exist a positive convolution kernel $K$ with $\sigma_{K}=\sigma_{*}$ and $\mu_{K}=\mu_{*}$. Indeed, unlike for $d=2$, not every positive function can arise as the mobility associated with a convolution kernel via equation (42), even if $\sigma_{*}$ satisfies the conclusion of Lemma 1. This can be seen with the following example: Take $\mu_{*}$ to be

$$
\mu_{*}(\theta, \phi)=\left(\mathcal{J}_{s}\left[1-2 \eta\left(\frac{\phi-\frac{1}{2}}{\delta}\right)\right]\right)^{-1}
$$

Choosing $\delta>0$ small enough, we see that $\mu_{*}(n)>0$ for all $n \in \mathbb{S}^{2}$. Assume that for some $K \geq 0$, we have $\mu_{K}=\mu_{*}$. Then, by (43) and the injectivity of $\mathcal{J}_{s}$, we have

$$
\int_{0}^{\infty} K(r, \theta, \phi) r^{3} d r=1-2 \eta\left(\frac{\phi-\frac{1}{2}}{\delta}\right) .
$$

The left hand side is always positive, but the right hand side is negative for $\phi \approx 0$, which is a contradiction.

The good news is that for the large and natural class of convex mobilities, it is possible to construct positive convolution kernels, as long as the surface tension satisfies the conclusion of Lemma 1. In $d=3$, we are thus led to the following assumptions on the pair $\left(\sigma_{*}, \mu_{*}\right)$ :

(2.1) $B_{\sigma_{*}}$ is strongly convex and $\partial B_{\sigma_{*}}$ is smooth,

(2.2) $W_{\sigma_{*}}$ is the dilation of a zonoid by a sphere,

(2.3) $\mu_{*}: \mathbb{S}^{2} \rightarrow \mathbb{R}^{+} \backslash\{0\}$ is smooth, and

(2.4) $\mu_{*}: \mathbb{R}^{3} \rightarrow \mathbb{R}^{+}$defined as $\mu_{*}(x)=|x| \mu_{*}\left(\frac{x}{|x|}\right)$ is convex.

It is worth repeating that condition (2.2) is essentially necessary, as explained above. It is also quite general, since it allows approximating any anisotropy that can arise as the continuum limit of a ferromagnetic Ising model; see e.g. the discussion in [13]. Furthermore, condition 4 is very natural: It merely stipulates that evolution (18) arises as 
gradient descent for the variational model (5) with respect to a possibly anisotropic norm on normal vector fields, as discussed in Section 2. We can now present our construction:

Proposition 4 Under conditions (2.1)-(2.4) on $\sigma_{*}$ and $\mu_{*}$, there exists a positive, smooth, compactly supported convolution kernel $K$ : $\mathbb{R}^{3} \rightarrow \mathbb{R}^{+}$such that $\sigma_{K}=\sigma_{*}$ and $\mu_{K}=\mu_{*}$.

Proof: As for $d=2$, we look for a kernel $K$ that in spherical coordinates has the form

$$
K(r, \theta, \phi)=\alpha(\theta, \phi) \eta(\beta(\theta, \phi) r)
$$

where $\alpha, \beta: \mathbb{R}^{2} \rightarrow \mathbb{R}^{+}$are $2 \pi$-periodic in each variable, smooth, and invariant under $(\theta, \phi) \rightarrow(\theta+\pi, \phi+\pi)$. Substituting (69) into (52) this time gives

$$
\begin{aligned}
\frac{\alpha(\theta, \phi)}{\beta^{4}(\theta, \phi)} m_{3} & =\omega_{*}(\theta, \phi), \text { and } \\
\frac{\alpha(\theta, \phi)}{\beta^{2}(\theta, \phi)} m_{1} & =\mathcal{J}_{s}^{-1}\left[\frac{1}{\mu_{*}}\right](\theta, \phi) .
\end{aligned}
$$

Hypothesis on $\sigma_{*}$ ensures that $\omega_{*}(\theta, \phi)>0$. Thanks again (as in Lemma 1) to Theorem 5.2 of [19],

$$
\mathcal{J}_{s}^{-1}\left[\frac{1}{\mu_{*}}\right]>0 \text {. }
$$

since our hypothesis on $\mu_{*}$ implies that $\frac{1}{\mu_{*}}$ is the radial function of a convex body with smooth boundary.

Solving for $\alpha(\theta, \phi)$ and $\beta(\theta, \phi)$ in (70), we get

$$
\begin{aligned}
\alpha(\theta, \phi) & =\frac{m_{3}}{m_{1}^{2}} \frac{\left(\mathcal{J}_{s}^{-1}\left[\frac{1}{\mu_{*}}\right]\right)^{2}}{\mathcal{J}_{s}^{-1} \square \sigma_{*}}, \text { and } \\
\beta(\theta, \phi) & =\frac{m_{3} \mathcal{J}_{s}^{-1}\left[\frac{1}{\mu_{*}}\right]}{m_{1} \mathcal{J}_{s}^{-1} \square \sigma_{*}}
\end{aligned}
$$

expressed in terms of standard transforms. Both are positive functions. Formulas (69) \& (72) provide an explicit prescription for the desired convolution kernel, which is positive.

Remark: In fact, in the language of e.g. [18, 19, 30], a mobility $\mu_{*}$ can arise from a positive convolution kernel in threshold dynamics algorithms if and only if $\frac{1}{\mu_{*}}$ is the radial function of an intersection body. However, this characterization is almost by definition of an intersection body (which is not as transparent as that of a zonoid), and therefore does not shed much light on the matter. 


\subsection{Kernels with Positive Fourier Transform}

It turns out that we can construct a smooth, rapidly decaying convolution kernel with positive Fourier transform, in any spatial dimension $d$, as long as the target surface tension $\sigma_{*}: \mathbb{S}^{d-1} \rightarrow \mathbb{R}^{+}$and the target mobility $\mu_{*}: \mathbb{S}^{d-1} \rightarrow \mathbb{R}^{+}$satisfy the minimal assumptions (1.1) \& (1.2) of Section 4.1.1. Our construction and resulting kernels are similar to those of [8], but are more general since we accommodate any mobility, whereas the kernels of [8] are restricted to the (important) special case $\mu_{*}=\sigma_{*}$. Moreover, the kernels of [8] are singular in the Fourier domain for all but ellipsoidal anisotropies, leading to slow decay in the physical domain. This technical issue is also easily rectified with our construction.

Recall that according to (44), we can find a kernel $K$ with the desired target surface tension and mobility by solving the system

$$
\left\{\begin{aligned}
\text { F. P. } & \int_{\mathbb{R}} \frac{\widehat{K}(n \xi)}{\xi^{2}} d \xi=-2 \pi \sigma_{*}(n), \text { and } \\
\int_{\mathbb{R}} \widehat{K}(n \xi) d \xi & =\frac{1}{2 \pi \mu_{*}(n)} .
\end{aligned}\right.
$$

This is particularly simple since the equations are pointwise in $n \in \mathbb{S}^{d-1}$ (unlike in the physical space construction of Section 4.1, as we shall see). We have:

Proposition 5 Under conditions (1.1) $\&(1.2)$ on $\sigma_{*}$ and $\mu_{*}$, there exists a convolution kernel $K: \mathbb{R}^{d} \rightarrow \mathbb{R}$ in Schwartz class and a constant $\gamma>0$ such that $\sigma_{K}=\sigma_{*}, \mu_{K}=\gamma \mu_{*}$, and $\widehat{K} \geq 0$.

Proof Let $\zeta: \mathbb{R} \rightarrow \mathbb{R}$ be a smooth function that satisfies the following conditions:

1. $\zeta(x) \geq 0$ and $\zeta(x)=\zeta(-x)$ for all $x$,

2. $\zeta(x)=0$ for $|x| \leq 1$,

3. $\zeta(x)=x^{2}$ for $|x| \geq 2$.

We want to construct a kernel $K$ such that $\widehat{K}$ will satisfy (73). Let $\alpha, \beta: \mathbb{R}^{d} \rightarrow[0, \infty]$ be one homogeneous functions. Then define the kernel $K$ by:

$$
\left.\widehat{K}(\xi)=\frac{1}{2} \exp (-\zeta(\alpha(\xi)))+\frac{1}{2} \exp (-\zeta(\beta(\xi)))\right) .
$$

Substituting (74) into (73) yields the following equations on $\alpha$ and $\beta$ in terms of the targets $\sigma_{*}$ and $\mu_{*}$ :

$$
\gamma \mu_{*}^{-1}(n)=\frac{1}{4 \pi} \int_{\mathbb{R}} \exp (-\zeta(\xi \alpha(n)))+\exp (-\zeta(\xi \beta(n))) d \xi
$$


and

$$
\sigma_{*}(n)=\frac{1}{4 \pi} \int_{\mathbb{R}} \frac{1-\exp (-\zeta(\xi \alpha(n)))}{\xi^{2}}+\frac{1-\exp (-\zeta(\xi \beta(n)))}{\xi^{2}} d \xi .
$$

Note that we have introduced a constant $\gamma$ to the mobility $\mu$ to ensure that a solution will exist. Let

$$
s_{0}=\frac{1}{4 \pi} \int_{\mathbb{R}} e^{-\zeta(\xi)} d \xi \text { and } s_{2}=\frac{1}{4 \pi} \int_{\mathbb{R}} \frac{1-e^{-\zeta(\xi)}}{\xi^{2}} d \xi .
$$

Then, with the changes of variables $\xi \rightarrow \xi \alpha(n)$ and $\xi \rightarrow \xi \beta(n)$, equations (75) and (76) become

$$
\frac{\gamma \mu_{*}^{-1}(n)}{s_{0}}=\frac{1}{\alpha(n)}+\frac{1}{\beta(n)},
$$

and

$$
\frac{\sigma_{*}(n)}{s_{2}}=\alpha(n)+\beta(n) .
$$

To simplify notation let $a(n)=\frac{\gamma \mu_{*}^{-1}(n)}{s_{0}}$ and $b(n)=\frac{\sigma_{*}(n)}{s_{2}}$. Eliminating $\beta(n)$ in $(77) \&(78)$ and rearranging leads to the following quadratic in $\alpha(n)$ :

$$
\alpha(n)^{2}-b(n) \alpha(n)+\frac{b(n)}{a(n)}=0 .
$$

Solving for $\alpha(n)$ in (79) and then for $\beta(n)$ in (78), we get

$$
\begin{aligned}
& \alpha(n)=\frac{1}{2 s_{2} \gamma^{\frac{1}{2}}}\left(\gamma^{\frac{1}{2}} \sigma_{*}+\sqrt{\gamma \sigma_{*}^{2}-4 s_{0} s_{2} \mu_{*} \sigma_{*}}\right) \\
& \beta(n)=\frac{1}{2 s_{2} \gamma^{\frac{1}{2}}}\left(\gamma^{\frac{1}{2}} \sigma_{*}-\sqrt{\gamma \sigma_{*}^{2}-4 s_{0} s_{2} \mu_{*} \sigma_{*}}\right)
\end{aligned}
$$

In order for both solutions to be real we need $b(n) \geq \frac{4}{a(n)}$. Therefore, we need to choose $\gamma$ such that $\gamma \geq \frac{4 s_{0} s_{2} \mu_{*}(n)}{\sigma_{*}(n)}$ for all $n$. We also do not want the discriminant to vanish, since this may introduce singularities into $\alpha(n), \beta(n)$ that are not present in the anisotropy and mobility. Indeed as long as the discriminant does not vanish $\alpha$ and $\beta$ will have the same differentiability properties as $\mu_{*}$ and $\sigma_{*}$. However, at the same time, we would like $\alpha(n)$ and $\beta(n)$ to be as close to each other as possible so that the kernel is easy to sample. This suggests that a good choice for $\gamma$ is

$$
\gamma=(1+\epsilon) \max _{n \in S^{d-1}} \frac{4 s_{0} s_{2} \mu_{*}(n)}{\sigma_{*}(n)}
$$


for some small $\epsilon>0$. Formulas (74), (77) \& (78) provide an explicit prescription for the desired kernel $K$.

As explained in [13], equations (51) along with the favorable smoothness and decay properties noted above imply that kernels (74) satisfy all the conditions for the consistency step of the convergence proof in [27]; we thus have the following as an immediate consequence:

Corollary 2 For essentially any given anisotropic surface tension and mobility pair, whether in two or three dimensions, there exists a Schwartz class convolution kernel using which threshold dynamics algorithm (1) E) (2) is consistent with the evolution law (18).

Although the sign changing character (in physical space) of the convolution kernels in this section (as well as in [8]) precludes an immediate proof of convergence (to the corresponding viscosity solution) in the mold of [27], numerical evidence does not indicate any adverse effects, at least in the two phase setting.

\section{$5 \quad$ Numerical Evidence}

We demonstrate the new kernel constructions of Section 4 on both two and multi-phase curvature flow problems, and in two dimensional cases compare against front tracking simulations. To compare with front tracking whenever possible, we focus mostly on regular behavior (i.e. no topological changes), but of course, as is well known, threshold dynamics methods shine when it comes to gracefully handling topology changes. (Experiment of Figure 6 clearly indicates that this major benefit of threshold dynamics is completely preserved by the new algorithms and constructions of the present paper). Section 5.1 is devoted to experiments with two-phase anisotropic flows, while Section 5.2 focuses on multi-phase anisotropic flows. We demonstrate the original threshold dynamics algorithm (1)-(2), as well as its recent, fully anisotropic, multiphase extensions Algorithm (89) \& (90) and Algorithm (91)-(95) that are recalled in the Appendix, using these kernels.

The front tracking algorithm used for two dimensional comparisons represents the interfaces via explicit parametrizations. As such, it is essentially a finite differences discretization for a coupled system of parabolic PDEs in one space dimension, and can thus yield very accurate benchmark results by choosing a very fine discretization. (A parametric approach can be taken to models of this paper also in 3D, see e.g. [5]. Unfortunately, topological changes are a serious difficulty with this approach, especially in $3 \mathrm{D}$, and a major motivation for methods that represent interfaces implicitly, such as that of this paper, or the phase field, or the level set method). We used explicit (forward 
Euler) time stepping to keep matters as simple as possible. In our implementation, triple junctions are common discretization points for the curves that meet at them. Their coordinates are updated not directly by the curvature flow PDE, but by iteratively optimizing the energy of the system with respect to these coordinates only, at every time step, much as described in [28]. This is how the Herring condition (22) is imposed at the junctions. As is well known, the parametrization of the curves can drift very far from an arc-length parametrization, resulting in very non-uniformly spaced points on the curves, adversely effecting the stability (CFL) condition [9]. Hence, as is customary in front tracking, we periodically reparametrize the curves by arc-length, though as seldomly as possible to prevent accumulation of errors caused by small but inevitable perturbation to the curves during the process (an alternative is the approach of [25], or of e.g. [5] that also works in 3D, which maintain a regular parametrization through tangential velocities).

A few comments are in order regarding implementation of threshold dynamics algorithms as well. The consistency calculations (truncation error analysis) carried out e.g. in $[27,39,13]$ reveal that in the twophase setting, one would expect an error $O(\delta t)$ as $\delta t \rightarrow 0$. Similar calculations in [38] indicate that in the presence of junctions, the error becomes $O(\sqrt{\delta t})$, which is easy to understand: the kernels have width $\sqrt{\delta t}$ and hence start sensing the junction at any point of comparable distance on the smooth interfaces. Although these are modest convergence rates, they can be easily improved e.g. by Richardson extrapolation demonstrated in [37], [38] to be effective on threshold dynamics schemes, with or without junctions. Other important improvements include implementation on adaptive grids while maintaining the efficiency of Fourier transform based convolutions, also developed and demonstrated in [38] to achieve excellent accuracy.

Since our focus in this study is primarily on developing and verifying the requisite theory that enables adapting threshold dynamics to contexts in which no version of it so far exists due to a lack of fundamental understanding, we work with essentially the most basic version of the algorithms, except for the following well-known and very simple implementation detail to enhance spatial resolution: The convolution step arising in each threshold dynamics algorithm considered - such as (1) of the original MBO scheme (1) \& (2) - yields a smooth level set function that can be used (via interpolation) to estimate what fraction of each grid cell is occupied by the evolving set at the next time step, which can then be used in representing the characteristic function of the set. The more involved improvements mentioned above, which are very important in practical applications of threshold dynamics, can of course also be implemented on the new algorithms and using the new kernels developed in this paper.

In all the examples, the computational domain is a discretization 
of $[0,1]^{d}$, with $d=2$ or 3 .

\subsection{Two-phase, anisotropic flows}

(a) As an initial, modest test of the proposed kernel constructions, consider the task of simulating anisotropic, two-phase curvature flow in 2D given by (19), with the following choice of surface tension and mobility:

$$
\sigma\left(x_{1}, x_{2}\right)=\sqrt{x_{1}^{2}+4 x_{2}^{2}} \text { and } \mu\left(x_{1}, x_{2}\right)=1 .
$$

Note that the corresponding Wulff shape is an ellipse in this case:

$$
W_{\sigma}=\left\{\left(x_{1}, x_{2}\right): x_{1}^{2}+\frac{1}{4} x_{2}^{2} \leq 1\right\} .
$$

The construction of Section 4.1 yields the following positive convolution kernel: $K$ given in polar coordinates by $(55)$ with

$$
\alpha(\theta)=\frac{m_{2}^{\frac{1}{2}}}{4 m_{0}^{\frac{3}{2}}}\left(1+3 \sin ^{2} \theta\right)^{\frac{3}{4}} \text { and } \beta(\theta)=\frac{m_{2}^{\frac{1}{2}}}{2 m_{0}^{\frac{1}{2}}}\left(1+3 \sin ^{2} \theta\right)^{\frac{3}{4}} .
$$

When $\eta$ in the definition (55) of $K$ is given by (53), the relevant moments (54) appearing in (85) are approximately

$$
m_{0} \approx 0.3403 \text { and } m_{2} \approx 0.3737 \text {. }
$$

Figure 1 shows the result of computation starting with a circle of radius $\frac{1}{4}$ as the initial condition, and ending at time $t=\frac{1}{80}$. The red curves are the result of threshold dynamics with the new kernels, with a spatial discretization of $256 \times 256$ and using 25 time steps of size $5 \cdot 10^{-4}$. Front tracking result, serving as our benchmark and shown in blue, used 128 points to discretize the curve and required 20480 time steps.

(b) A more interesting anisotropy for numerical exploration is

$$
\sigma\left(x_{1}, x_{2}, x_{3}\right)=\max \left\{\left|x_{1}\right|,\left|x_{2}\right|,\left|x_{3}\right|\right\}
$$

i.e. the $\ell^{\infty}$ norm that has as its Wulff shape the octahedron:

$$
W_{\sigma}=\left\{\left(x_{1}, x_{2}, x_{3}\right):\left|x_{1}\right|+\left|x_{2}\right|+\left|x_{3}\right| \leq 1\right\} .
$$

Consider this with e.g. the constant mobility $\mu=1$. Since $W_{\sigma}$ is not a zonoid, according to Theorem 1, there exists no positive convolution kernel using which threshold dynamics scheme (1) \& (2) can even approximate the corresponding flow. The new, fully general kernel construction of Section 5.1, however, easily yields a Schwartz class kernel $K$ with positive Fourier transform that is consistent with this 

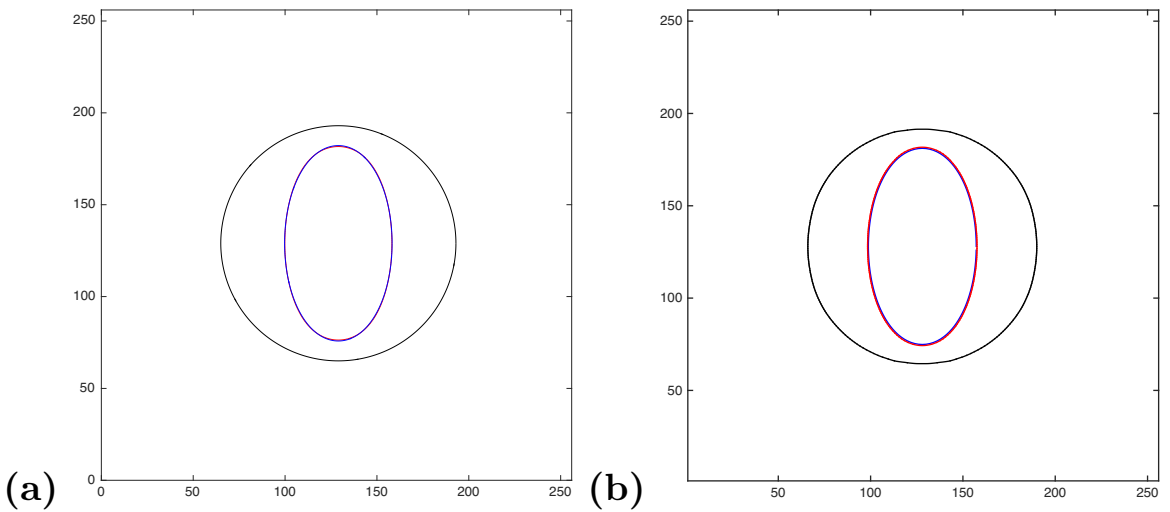

Figure 1: Evolution of an initial circle (black) under motion (19) with surface tension and mobility given by (83), computed using threshold dynamics algorithm (1) \& (2) and the convolution kernels from Sections 4.2 and 4.1 (red), compared against benchmark result obtained with front tracking (blue). (a) Using the convolution kernel with positive Fourier transform of Section 4.2. (b) Using the positive convolution kernel of Section 4.1.

choice of anisotropy and mobility. Figure 2 shows evolution of a cube under a volume preserving version [41,31] of Algorithm (1) \& (2) as implemented in [43] or [13] using the kernel construction of Section 5.1 using this anisotropy and mobility pair.

\subsection{Multi-phase, anisotropic flows}

Consider the initial three phase configuration shown in Figure 4 (a), subject to the following surface tension and mobility pairs:

$$
\begin{array}{rlrl}
\sigma_{1,2}\left(x_{1}, x_{2}\right) & =\sqrt{x_{1}^{2}+x_{2}^{2}} & \mu_{1,2}\left(x_{1}, x_{2}\right) & =1, \\
\sigma_{1,3}\left(x_{1}, x_{2}\right) & =\sqrt{\frac{1}{4} x_{1}^{2}+x_{2}^{2}}+\sqrt{x_{1}^{2}+\frac{1}{4} x_{2}^{2}} & \mu_{1,3}\left(x_{1}, x_{2}\right)=\frac{2 x_{1}^{2}+3 x_{2}^{2}}{4 \sqrt{x_{1}^{2}+x_{2}^{2}}} \\
\sigma_{2,3}\left(x_{1}, x_{2}\right) & =\sqrt{x_{1}^{2}+\frac{25}{16} x_{2}^{2}} & \mu_{2,3}\left(x_{1}, x_{2}\right) & =1 .
\end{array}
$$

The corresponding Wulff shapes for these anisotropies are shown in Figure 5.

The positive kernel construction given in Section 4.1 yields convolution kernels of the form

$$
K_{i, j}(r, \theta)=\alpha_{i, j}(\theta) \eta\left(r \beta_{i, j}(\theta)\right)
$$



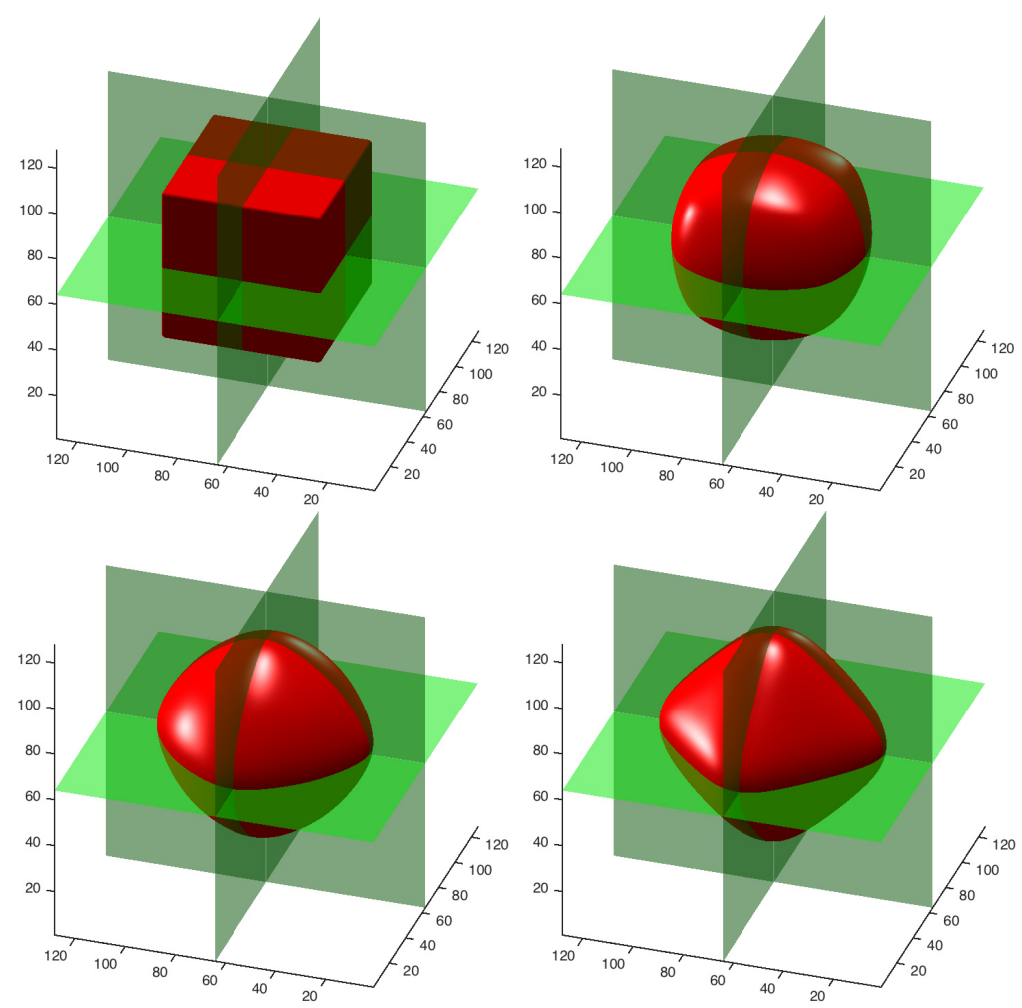

Figure 2: Evolution of a cube under volume preserving weighted mean curvature flow towards its Wulff shape the octahedron, with surface tension given by the $\ell^{\infty}$ norm and constant mobility. The corresponding kernel was obtained from the construction of Section 4.2. Compare with a similar experiment in [13] that uses a different convolution kernel that has the same surface tension but different mobility.

where, for example, $\alpha_{1,3}$ and $\beta_{1,3}$ are given by

$$
\begin{array}{r}
\alpha_{1,3}(\theta)=\frac{m_{2}^{\frac{1}{2}}}{2 m_{0}^{\frac{3}{2}}} \frac{\left(\cos ^{2}(\theta)+\frac{1}{4} \sin ^{2}(\theta)\right)^{\frac{3}{4}}\left(\frac{1}{4} \cos ^{2}(\theta)+\sin ^{2}(\theta)\right)^{\frac{3}{4}}}{\sqrt{\left(\cos ^{2}(\theta)+\frac{1}{4} \sin ^{2}(\theta)\right)^{\frac{3}{4}}+\left(\frac{1}{4} \cos ^{2}(\theta)+\sin ^{2}(\theta)\right)^{\frac{3}{4}}}} \\
\times\left(\frac{1}{2}+\frac{1}{4} \sin ^{2}(\theta)\right)^{-\frac{3}{2}}
\end{array}
$$


and

$$
\begin{array}{r}
\beta_{1,3}(\theta)=\frac{2 m_{2}^{\frac{1}{2}}}{m_{0}^{\frac{1}{2}}} \frac{\left(\cos ^{2}(\theta)+\frac{1}{4} \sin ^{2}(\theta)\right)^{\frac{3}{4}}\left(\frac{1}{4} \cos ^{2}(\theta)+\sin ^{2}(\theta)\right)^{\frac{3}{4}}}{\sqrt{\left(\cos ^{2}(\theta)+\frac{1}{4} \sin ^{2}(\theta)\right)^{\frac{3}{4}}+\left(\frac{1}{4} \cos ^{2}(\theta)+\sin ^{2}(\theta)\right)^{\frac{3}{4}}}} \\
\times\left(\frac{1}{2}+\frac{1}{4} \sin ^{2}(\theta)\right)^{-\frac{1}{2}}
\end{array}
$$

Figure 3 shows plots of these kernels.

(a)

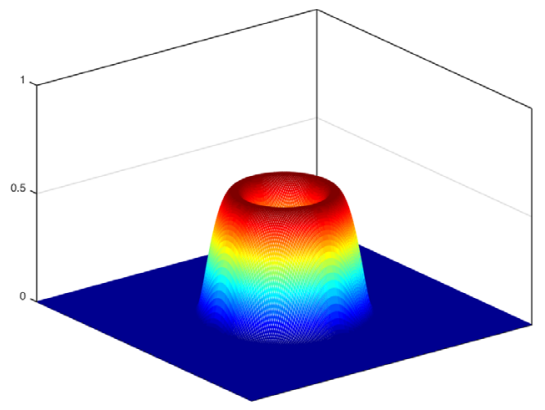

(b)

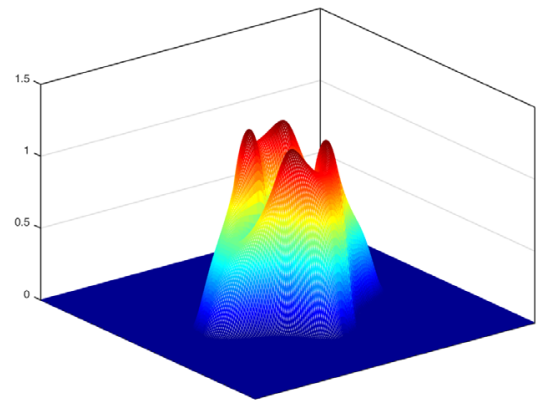

(c)

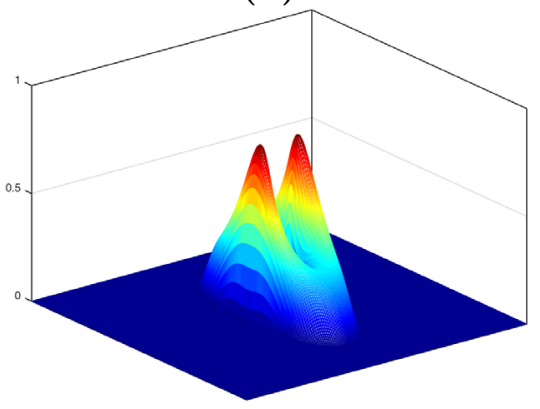

Figure 3: The kernels (a) $K_{1,2}$, (b) $K_{1,3}$, (c) and $K_{2,3}$ obtained from the construction of Section 4.1 for the surface tensions and mobilities used in the triple junction example of Figure 4.

Figure 4 (b) shows the resulting configuration at $t=0.01$ computed using these kernels in threshold dynamics Algorithm (89) \& (90) on a $256 \times 256$ grid with 20 time steps of size $\delta t=5 \cdot 10^{-4}$, and compared to the benchmark front tracking result computed using 200 grid points along each one of the three curves and 8000 time steps of size $\delta t=1.25 \cdot 10^{-6}$. There is very good agreement. We note that the kernels of Section 4.2 could have also been used in this example, since positivity of the convolution kernels or their Fourier transforms is sufficient for Algorithm (89) \& (90) to dissipate the multiphase nonlocal energy (34). Although numerical experiments with these kernels 
suggest convergence to the correct evolution, the error appears to be dramatically larger than that of using kernels of Section 4.1. We leave finding a more accurate version of the construction in Section 4.2 to a future study, and recommend kernels of Section 4.1 over them in the multiphase setting instead.

Finally, Figure 6 demonstrates how the seamless behavior through topological changes of the original threshold dynamics algorithm of [32] is entirely preserved by its extensions to the fully anisotropic, multiphase setting provided by Algorithms (89) \& (90) and (91)-(95). An initial condition consisting of 27 phases, obtained from a Voronoi construction for points chosen uniformly at random in a periodic domain of size $128 \times 128 \times 128$, is evolved via Algorithm (89) \& (90) using two different sets of surface tensions and mobilities: one in which all mobilities and surface tensions are equal and isotropic (where algorithm of [32] can be used), and another in which there is one distinguished phase, $i=1$, whose interfaces with other phases are anisotropic both in mobility and surface tension. Myriad topological changes occur on the surface of the "grain", as quadruple points collide and split, and existing facets are destroyed and new ones are formed. 


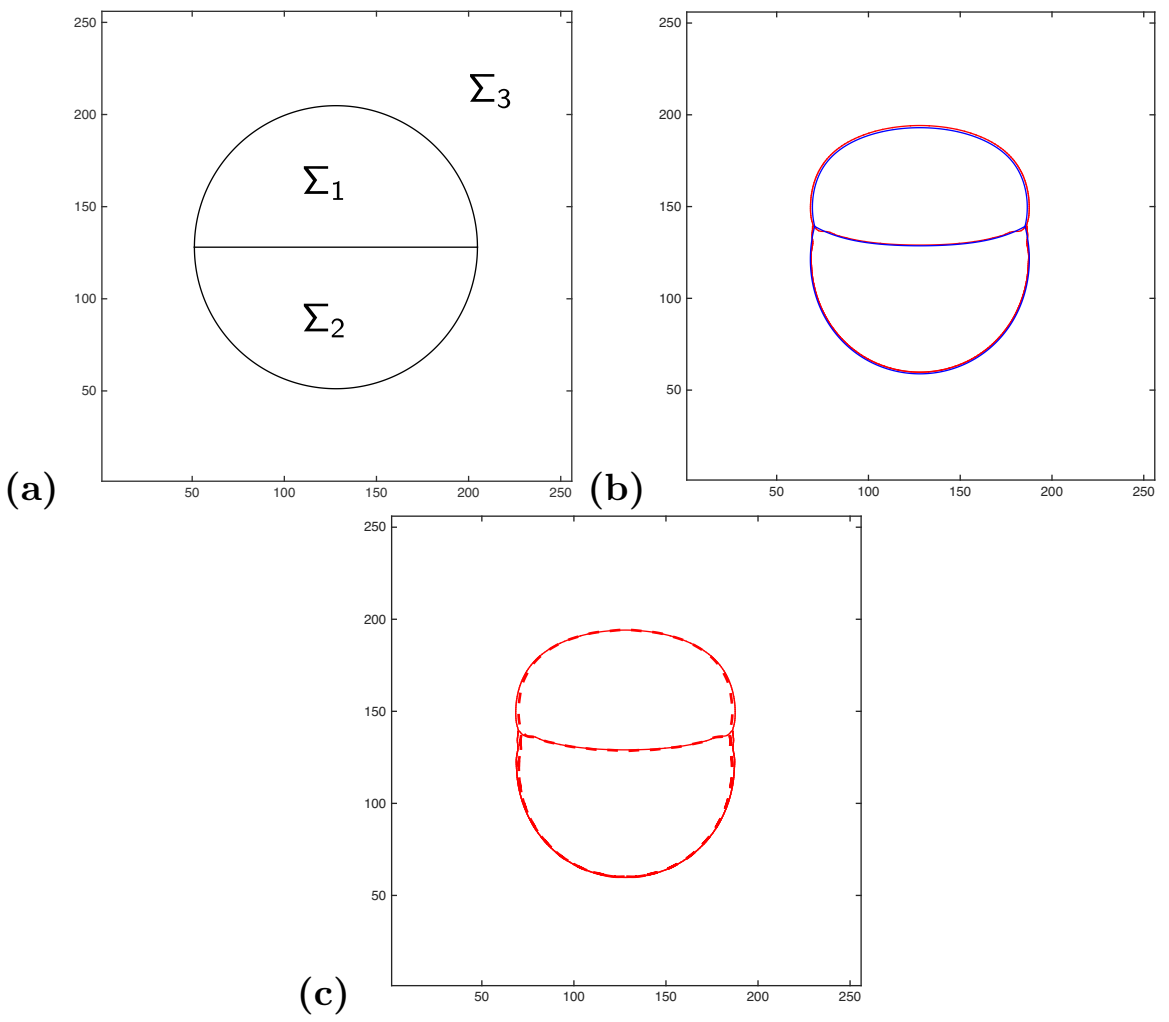

Figure 4: Evolution of a three-phase configuration with a pair of triple junctions under anisotropic curvature flow; each interface has a distinct prescribed surface tension (two of them anisotropic), two have constant mobility, and one has a normal dependent prescribed mobility, as described in detail in Section 5.2. (a) The initial condition. (b) Final configuration computed using threshold dynamics Algorithm (89) \& (90) and the positive kernel construction presented in Section 4.1 (red), compared to the benchmark result computed using front tracking (blue). (c) The same evolution computed using two different threshold dynamics algorithms: Algorithm (89) \& (90) shown in thin, solid vs. Algorithm (91)-(95) shown in thick, dashed line. Algorithm (89) \& (90) is faster, but Algorithm (91)-(95) has guaranteed unconditional gradient stability for essentially any collection of $N$-choose- 2 anisotropic surface tension and mobility pairs. 

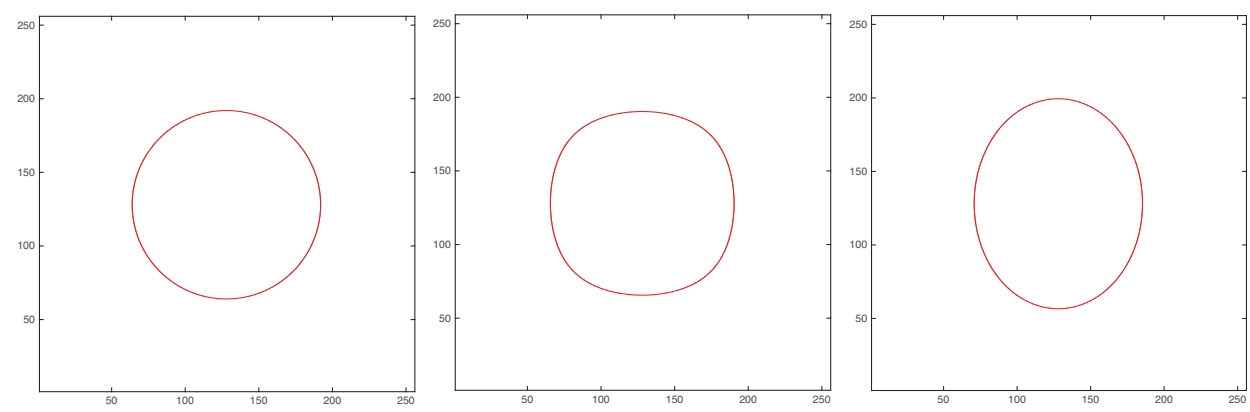

Figure 5: The Wulff shapes corresponding to the anisotropies used in the triple junction example. From left to right: $W_{\sigma_{1,2}}, W_{\sigma_{1,3}}$, and $W_{\sigma_{2,3}}$.
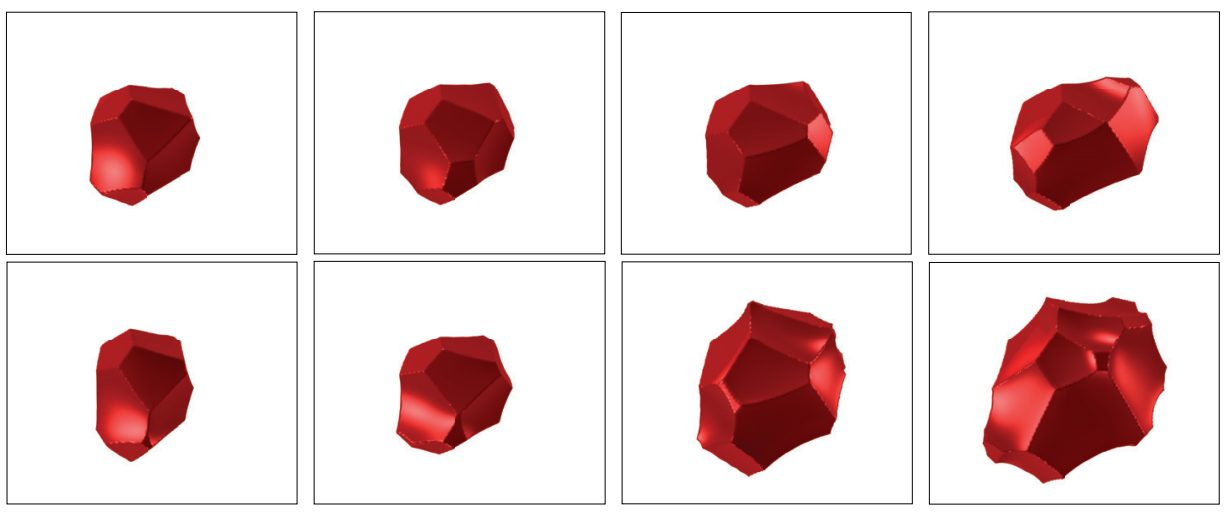

Figure 6: Evolution of phase $i=1$ out of a total of 27 phases, from two different simulations starting from the same initial condition. Top row: $\sigma_{i, j}(\theta, \phi)=\mu_{i, j}(\theta, \phi)=1$ for all $i \neq j$. Bottom row: $\sigma_{i, 1}(\theta, \phi)=1.1 \sqrt{1+0.3 \cos ^{2} \phi}$ and $\mu_{i, 1}(\theta, \phi)=\frac{1}{1.1} \sqrt{1+0.3 \cos ^{2} \phi}$ for $i \neq 1$, and $\sigma_{i, j}(\theta, \phi)=\mu_{i, j}(\theta, \phi)=1$ for $i \neq 1$ and $j \notin\{i, 1\}$. Many topological changes occur on the surface of the "grain", where quadruple points can be seen to collide and split numerous times.

\section{Appendix}

We recall from [13] and [14] extensions of the isotropic but unequal surface tension, multi-phase version of threshold dynamics Algorithm (37) \& (38) that was originally obtained in [15] to the fully anisotropic, multi-phase setting. Let the surface tensions $\sigma_{i, j}: \mathbb{S}^{d-1} \rightarrow \mathbb{R}^{+}$and mobilities $\mu_{i, j}: \mathbb{S}^{d-1} \rightarrow \mathbb{R}^{+}$be given. Let $K_{i, j}$ be convolution kernels that have these surface tensions and mobilities according to formulas 
(39) \& (42) constructed, for example, by one of the methods presented in Sections 4.2 and 4.1.

We start with the following completely straight forward generalization of Algorithm (37) \& (38), from [13]:

Algorithm: (from [13]) Given a time step size $\delta t>0$, alternate the following steps:

1. Convolution:

$$
\psi_{i}^{k}=\sum_{j \neq i}\left(K_{i, j}\right)_{\sqrt{\delta t}} * \mathbf{1}_{\Sigma_{j}^{k}} .
$$

2. Thresholding:

$$
\Sigma_{i}^{k+1}=\left\{x: \psi_{i}^{k}(x) \leq \min _{j \neq i} \psi_{j}^{k}(x)\right\} .
$$

Although Algorithm (89) \& (90) works well in practice, as shown in numerical experiments of [13] as well as Section 5.2 of this study, it appears difficult to establish that it dissipates the corresponding nonlocal energy (49). In [14], slightly more expensive variants of (89) \& (90) along the lines of Algorithm (45)-(48) are proposed that ensure energy dissipation at the expense of a greater number of convolution operations per time step. The added complexity does not scale with the number of grid points, but with the number of partitions, and thus remains manageable. We recall one of these, Algorithm (91)-(95), below. According to Proposition 4.5 in [14], it dissipates energy (49) for a very wide class of kernels that includes any kernel constructed in Section 4 of this work. Together, the algorithm and the new convolution kernels thus provide an unconditionally stable numerical scheme for the multiphase anisotropic energy (7) and its gradient descent dynamics (18) \& (22) at essentially full generality. 
Algorithm: Given an initial partition $\boldsymbol{\Sigma}^{0}=\left\{\Sigma_{i}^{0}\right\}_{i=1}^{N}$, the $(k+$ 1) $)^{t h}$ iteration $\boldsymbol{\Sigma}^{k+1}$ is obtained from $\boldsymbol{\Sigma}^{k}$ by a series of substeps indexed by $(m, n) \in \mathcal{I}_{N}$ For $(m, n) \neq(1,2)$ let $p(m, n)$ denote the predecessor of $(m, n)$ in the dictionary ordering of $\mathcal{I}_{N}$ and define $\boldsymbol{\Sigma}^{k, p(1,2)}=\boldsymbol{\Sigma}^{k}$ and $\boldsymbol{\Sigma}^{k,(N, N-1)}=\boldsymbol{\Sigma}^{k+1}$. Then $\boldsymbol{\Sigma}^{k,(m, n)}$ is obtained from $\boldsymbol{\Sigma}^{k, p(m, n)}$ as follows:

1. For each $(i, j) \in \mathcal{I}_{N}$ form the convolutions:

$$
\psi_{i, j}^{k,(m, n)}(x)=\left(K_{i, j}\right)_{\sqrt{\delta t}} * \mathbf{1}_{\Sigma_{j}^{k, p(m, n)}}
$$

2. For each $i$ form the sums:

$$
\Psi_{i}^{k,(m, n)}(x)=\sum_{j \neq i} \psi_{(i, j)}^{k,(m, n)}(x)
$$

3. Threshold the $m^{\text {th }}$ function:

$$
G^{k,(m, n)}=\left\{x \in D: \min _{i} \Psi_{i}^{k,(m, n)}(x)=\Psi_{m}^{k,(m, n)}(x)\right\}
$$

4. Grow set $m$ into set $n$ only:

$$
\Sigma_{m}^{k,(m, n)}=\Sigma_{m}^{k, p(m, n)} \cup\left(G^{k,(m, n)} \cap \Sigma_{n}^{k, p(m, n)}\right)
$$

5. Update set $n$ :

$$
\Sigma_{n}^{k,(m, n)}=\Sigma_{n}^{k,(m, n)} \backslash\left(G^{k,(m, n)} \cap \Sigma_{n}^{k, p(m, n)}\right)
$$

Let us also quote the following statement from [14] that concerns the stability of Algorithm (91)-(95):

Proposition 6 (from [14]) Let each convolution kernel $K_{i, j}$ be of the form $K_{i, j}=K_{i, j}^{1}+K_{i, j}^{2}$, with $K_{i, j}^{1} \geq 0$ and $\widehat{K_{i, j}^{2}} \geq 0$. Then, Algorithm (91)-(95) dissipates energy (49) at every time step.

Finally, note that all the new kernel constructions presented in this paper satisfy the conditions of Proposition 6 .

\section{Conclusions}

We have presented two simple and practical methods to construct convolution kernels to be used in threshold dynamics schemes for curvature motion in two or three dimensions. Our constructions allow, for the first time, specifying a possibly anisotropic surface tension and possibly anisotropic mobility simultaneously: these are encoded into the 
convolution kernel. Combining the new kernel constructions with extensions of threshold dynamics algorithms presented in [14], we arrived at unconditionally gradient stable schemes for fully anisotropic, multiphase motion by weighted mean curvature of networks in two and three dimensions. This level of generality allows specifying $n$-choose- 2 anisotropic surface tensions and $n$-choose- 2 anisotropic mobilities for a network of $n$ phases. Numerical experiments indicate that in the multiphase setting, one of our new kernel constructions gives significantly more accurate results than the other.

Along the way, our work has also elucidated necessary and sufficient conditions for the positivity of the convolution kernel used in a threshold dynamics scheme in terms of the surface tension and mobility factors of the desired weighted mean curvature flow.

\section{Acknowledgments}

Selim Esedoḡlu and Matt Jacobs were supported by NSF DMS-1317730. Pengbo Zhang was supported by a Department of Mathematics International Student Fund. Part of the work of Selim Esedoḡlu was carried out during a visit to Centre de Recherches Mathematiques and McGill University in Montreal. The hospitality of these institutions is gratefully acknowledged.

\section{References}

[1] G. Alberti and G. Bellettini. A non-local anisotropic model for phase transitions: asymptotic behavior of rescaled energies. European J. Appl. Math., 9:261-284, 1998.

[2] F. Almgren, J. E. Taylor, and L.-H. Wang. Curvature-driven flows: a variational approach. SIAM Journal on Control and Optimization, 31(2):387-438, 1993.

[3] L. Ambrosio and A. Braides. Functionals defined on partitions in sets of finite perimeter II: semicontinuity, relaxation, and homogenization. J. Math. Pures et Appl., 69:307-333., 1990.

[4] G. Barles and C. Georgelin. A simple proof of convergence for an approximation scheme for computing motions by mean curvature. SIAM J. Numer. Anal., 32:484-500, 1995.

[5] J. W. Barrett, H. Garcke, and R. Nürnberg. Parametric approximation of surface clusters driven by isotropic and anisotropic surface energies. Interfaces and Free Boundaries, 12:187-234, 2010.

[6] G. Bellettini and M. Paolini. Anisotropic motion by mean curvature in the context of Finsler geometry. Hokkaido Mathematical Journal, 25:537-566, 1996. 
[7] E. D. Bolker. A class of convex bodies. Transactions of the American Mathematical Society, 145:323-345, 1969.

[8] E. Bonnetier, E. Bretin, and A. Chambolle. Consistency result for a non-monotone scheme for anisotropic mean curvature flow. Interfaces and Free Boundaries, 14(1):1-35, 2012.

[9] L. Bronsard and B. Wetton. A numerical method for tracking curve networks moving with curvature motion. Journal of Computational Physics, 120(1):66-87, 1995.

[10] H. Busemann and C. M. Petty. Problems on convex bodies. Mathematica Scandinavica, 4:88-94, 1956.

[11] E. De Giorgi. New problems on minimizing movements. In C. Baiocchi and J. L. Lions, editors, Boundary value problems for PDE and applications, pages 81-98. Masson, 1993.

[12] A. di Carlo, M. E. Gurtin, and P. Podio-Guidugli. A regularized equation for anisotropic motion-by-curvature. SIAM Journal on Applied Mathematics, 52(4):1111-1119, 1992.

[13] M. Elsey and S. Esedoḡlu. Threshold dynamics for anisotropic surface energies. Technical report, University of Michigan, April 2016. Under review.

[14] S. Esedoḡlu and M. Jacobs. Convolution kernels and stability of threshold dynamics methods. Technical report, University of Michigan, August 2016. Under review.

[15] S. Esedoğlu and F. Otto. Threshold dynamics for networks with arbitrary surface tensions. Communications on Pure and Applied Mathematics, 68(5):808-864, 2015.

[16] L. C. Evans. Convergence of an algorithm for mean curvature motion. Indiana University Mathematics Journal, 42:553-557, 1993.

[17] C. Garcia-Cardona, E. Merkurjev, A. L. Bertozzi, A. Flenner, and A. G. Percus. Multiclass data segmentation using diffuse interface methods on graphs. IEEE Transactions on Pattern Analysis and Machine Intelligence, 36(8):1600-1613, 2014.

[18] R. J. Gardner. Intersection bodeis and the Busemann-Petty problem. Transactions of the American Mathematical Society, 342(1):435-445, 1994.

[19] R. J. Gardner. A positive answer to the Busemann-Petty problem in three dimensions. Annals of Mathematics, 140:435-447, 1994.

[20] Paul Goodey and Wolfgang Weil. Centrally symmetric convex bodies and the spherical Radon transform. Journal of Differential Geometry, 35:675-688, 1992.

[21] G. Gottstein and L. S. Shvindlerman. Grain boundary migration in metals. CRC Press, 2nd edition, 2010. 
[22] S. Helgason. The Radon Transform, volume 5 of Progress in Mathematics. Birkhauser, Boston, MA, 1999.

[23] C. Herring. The Physics of Powder Metallurgy, chapter Surface tension as a motivation for sintering, pages 143-179. McGraw Hill, 1951.

[24] E. A. Holm, G. N. Hassold, and M. A. Miodownik. Dimensional effects on anisotropic grain growth. In G. Gottstein and D. Molodov, editors, Recrystallization and Grain Growth, pages 239-244, RWTH Aachen, Germany, August 2001. First Joint International Conference on Recrystallization and Grain Growth, Springer-Verlag.

[25] T. Y. Hou, J. S. Lowengrub, and M. J. Shelley. Removing the stiffness from interfacial flows with surface tension. Journal of Computational Physics, 114(2):312-338, 1994.

[26] F. J. Humphreys and M. Hatherly. Recrystallization and related annealing phenomena. Elsevier, 2004.

[27] H. Ishii, G. E. Pires, and P. E. Souganidis. Threshold dynamics type approximation schemes for propagating fronts. Journal of the Mathematical Society of Japan, 51:267-308, 1999.

[28] D. Kinderlehrer, I. Livshitz, and S. Taasan. A variational approach to modeling and simulation of grain growth. SIAM Journal on Scientific Computing, 28(5):1694-1715, 2006.

[29] S. Luckhaus and T. Sturzenhecker. Implicit time discretization for the mean curvature flow equation. Calculus of Variations and Partial Differential Equations, 3(2):253-271, 1995.

[30] E. Lutwak. Intersection bodies and dual mixed volumes. Advances in Mathematics, 71:232-261, 1988.

[31] P. Mascarenhas. Diffusion generated motion by mean curvature. CAM Report 92-33, UCLA, July 1992. (URL = http://www.math.ucla.edu/applied/cam/index.html).

[32] B. Merriman, J. K. Bence, and S. Osher. Motion of multiple junctions: a level set approach. Journal of Computational Physics, 112(2):334-363, 1994.

[33] B. Merriman, J. K. Bence, and S. J. Osher. Diffusion generated motion by mean curvature. In J. Taylor, editor, Proceedings of the Computational Crystal Growers Workshop, pages 73-83. AMS, 1992.

[34] M. Miranda, D. Pallara, F. Paronetto, and M. Preunkert. Shorttime heat flow and functions of bounded variation in $\mathbb{R}^{N}$. Ann. Fac. Sci. Toulouse, Mathematiques, 16(1):125-145, 2007.

[35] W. W. Mullins. Two dimensional motion of idealized grain boundaries. J. Appl. Phys., 27:900-904, 1956. 
[36] D. Mumford and J. Shah. Optimal approximations by piecewise smooth functions and associated variational problems. Communications on Pure and Applied Mathematics, 42:577-685, 1989.

[37] S. J. Ruuth. Efficient algorithms for diffusion-generated motion by mean curvature. PhD thesis, The University of British Columbia, 1996.

[38] S. J. Ruuth. A diffusion generated approach to multiphase motion. Journal of Computational Physics, 145:166-192, 1998.

[39] S. J. Ruuth. Efficient algorithms for diffusion-generated motion by mean curvature. Journal of Computational Physics, 144:603-625, 1998.

[40] S. J. Ruuth and B. Merriman. Convolution generated motion and generalized Huygens' principles for interface motion. SIAM Journal on Applied Mathematics, 60:868-890, 2000.

[41] S. J. Ruuth and B. Wetton. A simple scheme for volumepreserving motion by mean curvature. Journal of Scientific Computing, 19(1):373-384, 2003.

[42] J. E. Taylor. Overview no. 98: Mean-curvature and weighted mean-curvature. Acta Metallurgica et Materialia, 40(7):1475$1485,1992$.

[43] X. Xu, D. Wang, and X. Wang. An efficient threshold dynamics method for wetting on rough surfaces. arXiv:1602.04688, February 2016. 